\title{
Modeling extracellular matrix viscoelasticity using smoothed particle hydrodynamics with improved boundary treatment
}

\author{
Tommy Heck ${ }^{\mathrm{a}}$, Bart Smeets ${ }^{\mathrm{b}}$, Simon Vanmaercke ${ }^{\mathrm{b}}$, Pinaki Bhattacharya ${ }^{\mathrm{a}}$, \\ Tim Odenthal ${ }^{\mathrm{b}}$, Herman Ramon ${ }^{\mathrm{b}}$, Hans Van Oosterwyck ${ }^{\mathrm{a}, \mathrm{c}, \mathrm{e}, *}$, Paul Van \\ Liedekerke $^{\mathrm{d}, \mathrm{e}}$ \\ ${ }^{a}$ Biomechanics Section, KU Leuven, Celestijnenlaan 300C, 3001 Heverlee, Belgium \\ ${ }^{b}$ MeBioS, KU Leuven Kasteelpark Arenberg 30, 3001 Heverlee, Belgium \\ ${ }^{c}$ Prometheus, Division of Skeletal Tissue Engineering, KU Leuven, Herestraat 49, 3000 \\ Leuven, Belgium \\ ${ }^{d}$ INRIA Paris, 2 Rue Simone IFF, 75012 Paris, France \\ ${ }^{e}$ Joint last author
}

\begin{abstract}
We propose viscoelastic smoothed particle hydrodynamics (SPH) with extended boundary conditions as a new method to model the extracellular matrix (ECM) in contact with a migrating cell. The contact mechanics between a cell and ECM is modeled based on an existing boundary method in SPH that corrects for the well-known missing kernel support problem in Fluid Structure Interactions (FSI). This boundary method is here extended to allow the modeling of moving boundaries in contact with a viscoelastic solid. To validate the method, simulations are performed of tractions applied to a viscoelastic solid, Stokes flow around an array of square pillars, and indentation of a viscoelastic material with a circular indenter. By drop out of the inertial terms in the SPH equations of motion, the new SPH formulation allows to solve problems in a low Reynolds environment with a timestep independent of the particle spacing, permitting to model processes at the cellular scale (i.e. $\mu \mathrm{m}$-scale). The potential of the method to capture cell-ECM interactions is demonstrated by simulation of a self propelling object that locally degrades the ECM by fluidizing it to permit
\end{abstract}

*Corresponding author: hans.vanoosterwyck@kuleuven.be; Celestijnenlaan 300C - box 2419, 3001 Leuven, Belgium

Preprint submitted to Computer Methods in Applied Mechanics and Engineering May 12, 2017 
migration. This should enable us to model and understand realistic cell-matrix interactions in the future.

Keywords: smoothed particle hydrodynamics, boundary conditions, viscoelastic, extracellular matrix, degradation, cell migration

2015 MSC: 00-01, 99-00

\section{Introduction}

Cell mechanics plays an important role in the regulation of many biological processes. In processes such as morphogenesis, wound healing, cancer cell migration and angiogenesis, the ability of cells to sense their environment and to migrate is vital. Cells are surrounded by extracellular matrix (ECM), which is an organized network of molecules secreted by the cells that provides among other the structural support for the tissue. During migration, cells adhere to the ECM, generate protrusive and contractile forces and degrade the ECM. At the same time, the forces generated between the cell and its environment affect the behavior of a cell through mechanotransduction, the mechanism by which cells transform a mechanical stimulus into a chemical response. Therefore, a good understanding of the forces applied by cells to other cells and the surrounding ECM is vital in the study of many biological processes and pathologies.

In order to improve our understanding of the role of ECM mechanics for cell migration, computational models are being developed (e.g. [1, 2, 3]). A computational model of the ECM surrounding a cell should be able to capture the characteristics of cell-ECM interactions, i.e. large deformations, ECM degradation and the movement of cells through the ECM. Models have been published in which the ECM is represented by a coarse grained node-spring network [3], allowing to model the migration of a cell model through the ECM by local removal of springs and nodes. However, such coarse grained node-spring networks may not correctly represent the appropriate constitutive equations and can introduce artifacts depending on the neighbor topology of the nodes in the network. Continuum methods like finite element (FE) methods offer a possibil- 
ity to model the mechanics of the ECM $(4,5,6,7])$, but might run into problems when large deformations or material degradation are present, requiring computationally expensive remeshing procedures. The arbitrary Lagrangian-Eulerian (ALE) method or the immersed boundary method could be used to deal with moving boundaries and large deformations of the ECM. For example, MacKenzie et al. used the ALE FE method to model the reaction-diffusion system of a ligand around a deforming cell membrane representation [8]. Guyot et al. used the immersed boundary method to investigate the effect of microscopic flow on deformable cell models attached to a 3D scaffold in a perfusion bioreactor 9 . However, these models describe fluid-solid interaction at a moving boundary instead of solid-solid interaction. A similar implementation using ALE or the immersed boundary method has not been published yet on migration of a cell through an ECM which requires solid-solid interaction and degradation of the ECM. Meshless particle based methods can be a valuable alternative here because they can deal with deformable interfaces ([10]), large deformations and discontinuities, while at the same time allowing a natural and efficient coupling with other particle methods such as the Discrete Element Method which can realistically model deformable cells as individual, discrete objects ([11, 12, 13]). To the best of our knowledge, no computational model has been published that captures cell migration through a continuous ECM that functions as a mechanical obstacle for the cell and requires degradation for the cell to pass through.

In this paper, smoothed particle hydrodynamics ( $\mathrm{SPH}$ ) is proposed as a model for the ECM, in particular for homogeneous non-fibrillar matrices that can be used for cell encapsulation culturing, like polysaccharides and polyethylene glycol (PEG) gels. SPH is a meshless Lagrangian numerical method developed initially for the modeling of gas dynamics in astrophysical problems and later used mainly for modeling of fluids [14, 15]. In SPH, a material is divided into a set of discrete elements, called particles, for which the material properties (e.g. mass, density, velocity and hydrostatic pressure) are described. By using a smoothing kernel to approximate these properties for the next time step based on information of the surrounding particles, the continuum laws of fluid and 
solid mechanics are implemented in a discrete manner. SPH is known to have good conservation properties and enables to implement continuum mechanical, constitutive laws independent of the particle discretization (which is more problematic with other particle-based methods like the Discrete Element Method). Besides, the Lagrangian character of SPH allows to naturally capture discrete processes in a continuum material. SPH is in general computationally more expensive than mesh-based methods, although the latter may require remeshing in order to deal with (very) large deformations, thereby reducing their efficiency. Besides, the treatment of boundary conditions can be troublesome in SPH and $\mathrm{SPH}$ is known to have a limiting second-order convergence rate for increasing number of particles [16]. Recently, Das and Cleary demonstrated that SPH is a robust and suitable method for accurate modeling of elastic solids under compression in $2 \mathrm{D}$ and provides solutions that agree well with those of the FE method [17].

Recently, a method has been introduced for flow problems at low Reynolds numbers that allows the use of a timestep which is orders of magnitude higher than that in SPH. This higher time step is required to model cellular processes at the time scale of hours to days. In this method, called non-inertial SPH (NSPH), the low Reynolds number system is assumed to be overdamped. This permits to neglect the inertial term and thereby the reduction of the problem to a first order system from which velocities can be calculated directly [18. NSPH has been shown to reproduce the results obtained with SPH for a number of standard fluid dynamics problems and for more complex problems like the movement of a red blood cell in plasma, thus demonstrating the capabilities of this method. Since cell mechanics takes place in low Reynolds number environments because of the small length scale (i.e. $\mu \mathrm{m}$-scale) [19], NSPH could provide a suitable method to model ECM mechanics and degradation. In this paper we introduce for the first time NSPH for viscoelastic solid behavior and compare its accuracy with viscoelastic SPH [20, 21].

One of the most challenging parts of SPH is to treat boundary conditions correctly. This difficulty comes from the fact that the SPH formulation is based 
on the assumption that the smoothing kernel applied is completely occupied by neighboring particles. However, particles close to a rigid boundary miss a part of the kernel support and therefore the error in calculating hydrostatic pressure and deviatoric stress is largest for these particles. Since the cell-ECM boundary is an important regulator for cell adhesion and force generation and since the force and displacement fields near the cell-ECM boundary are most interesting from a cellular mechanics point of view, an adequate treatment of these boundary conditions is necessary. As has been outlined by Ferrand et al., various methods have been used to implement boundary conditions of which repulsive forces and fictitious particles are used most often [22]. Repulsive forces such as forces based on the Lennard-Jones potential are very easy to implement, but often lead to spurious effects due to the larger error that is made in calculation of hydrostatic pressure near the boundary. The use of fictitious particles behind a predefined boundary prevents this spurious behavior, but it is challenging to position these particles for a boundary with a complex geometry, and the situation is made worse for a moving and deforming boundary such as that of cell. A better method to implement boundary conditions is the method introduced by Kulasegaram et al. and further improved by Ferrand et al. in which a correction is applied to the SPH formulation based on the missing kernel area support near the boundary [22, 23]. This method however was only implemented for the contact between a fluid and a stationary wall. Here, this method is extended by introducing for the first time proper boundary conditions for contact between (N)SPH models of a viscous fluid and a viscoelastic solid on the one hand, and a stationary or moving boundary on the other hand. We demonstrate that errors are introduced by this method when the material approaches a boundary and propose a contact criterion to reduce these errors.

This paper is organized as follows. First we introduce the formulations for nearly incompressible viscous fluid and viscoelastic solid behavior in 2D SPH and NSPH. Then we introduce the extended method to model boundary conditions between a viscoelastic solid and a rigid moving wall. We perform simulations of tractions applied to a viscoelastic solid material and compare 
the accuracy of the viscoelastic NSPH method with SPH, as well as with FE simulations that serve as a reference. In order to demonstrate the boundary method for both viscous fluid and viscoelastic solids, we show the accuracy of the boundary method with a simulation of Stokes flow around an array of rigid square pillars and a simulation of indentation of a viscoelastic solid with a rigid, yet moving circular indenter. For the latter again results are compared to FE simulations. Finally, to illustrate the future capabilities of the method, we run simulations of a simplified cell-ECM interaction and migration process, whereby a cell (modeled as a rigid, self propelling object) locally degrades a viscoelastic ECM and applies forces to it in order to be able to move through it.

\section{Methods}

\subsection{Basic SPH formulation}

In SPH a material is represented as a set of mass points called particles that carry properties as density, pressure and velocity. The information of neighboring particles $j$ is used to approximate function values $A_{i}$ and their derivatives $\nabla_{i} A_{i}$ at a specific particle $i$ by discretized convolution with a smoothing kernel $W$ :

$$
\left\langle A_{i}(\boldsymbol{r})\right\rangle \equiv \sum_{j} m_{j} \frac{A_{j}}{\rho_{j}} W\left(\left|\boldsymbol{r}_{i j}\right|, h\right)
$$

and

$$
\left\langle\nabla_{i} A_{i}(\boldsymbol{r})\right\rangle \equiv \sum_{j} m_{j} \frac{A_{j}}{\rho_{j}} \nabla_{i} W\left(\left|\boldsymbol{r}_{i j}\right|, h\right),
$$

with $A_{j}, m_{j}$ and $\rho_{j}$ the function value, mass and density of the neighboring particle $j$ within the kernel support. The value of the smoothing kernel $W_{i j}=W\left(\left|\boldsymbol{r}_{i j}\right|, h\right)$ for particle $j$ depends on the distance between both particles $\boldsymbol{r}_{i j}$ and the smoothing length $h$. The notation $\boldsymbol{r}_{i j}=\boldsymbol{r}_{i}-\boldsymbol{r}_{j}$ will also be used in the same manner for other vectors later on. The derivative of the smoothing kernel $\boldsymbol{\nabla}_{i} W_{i j}=\boldsymbol{\nabla}_{i} W\left(\left|\boldsymbol{r}_{i j}\right|, h\right)$ is used to approximate the derivative of the function value, with $\nabla_{i}$ the gradient with respect to the coordinates 
of particle $i$. Several interpolation kernels have been used before, of which the cubic spline, quintic spline and Wendland kernel (which has the advantage that it prevents particle clustering) have been used frequently [24, 25]. These kernels all decrease monotonically, are continuous, have a continuous derivative and they have a finite, compact support.

\subsection{Nearly incompressible Newtonian fluid}

Eq. (1) and Eq. (2) are used to discretize the continuum laws of fluid and solid mechanics [24]. The conservation of mass is implemented as:

$$
\frac{\mathrm{d} \rho_{i}}{\mathrm{~d} t}=\sum_{j} m_{j} \boldsymbol{v}_{i j} \cdot \nabla_{i} W_{i j}
$$

with $\boldsymbol{v}$ the particle velocity. The conservation of momentum for a nearly incompressible fluid is modeled as:

$$
m_{i} \frac{\mathrm{d} \boldsymbol{v}_{i}^{v}}{\mathrm{~d} t}=\boldsymbol{F}_{i}^{p}+\boldsymbol{F}_{i}^{v}+\boldsymbol{F}_{i}^{b},
$$

with $\boldsymbol{F}_{i}^{p}$ the pressure forces, $\boldsymbol{F}_{i}^{v}$ the viscous forces and $\boldsymbol{F}_{i}^{b}$ body forces. The pressure forces include the effect of volume changes due to internal pressure:

$$
\boldsymbol{F}_{i}^{p}=-m_{i} \sum_{j} m_{j}\left(\frac{p_{i}}{\rho_{i}^{2}}+\frac{p_{j}}{\rho_{j}^{2}}\right) \nabla_{i} W_{i j},
$$

with $p$ the hydrostatic pressure. For the viscous forces multiple formulations have been proposed before [24, 26]. Here, the formulation of Morris et al. will be used [26]:

$$
\boldsymbol{F}_{i}^{v}=m_{i} \sum_{j} m_{j} \frac{\mu_{i}+\mu_{j}}{\rho_{i} \rho_{j}} \frac{\boldsymbol{r}_{i j} \cdot \nabla_{i} W_{i j}}{\left|\boldsymbol{r}_{i j}\right|^{2}+\eta^{2}} \boldsymbol{v}_{i j}
$$

with $\mu$ the dynamic viscosity of the fluid and $\eta=0.01 h^{2}$ a correction factor that prevents singularity when particles approach each other. 
The equation of state that defines the relationship between pressure and density of the particles is:

$$
p_{i}=p_{0}+K\left(\left(\frac{\rho_{i}}{\rho_{0}}\right)^{\xi}-1\right),
$$

where $\rho_{0}$ and $p_{0}$ are the initial density and pressure and $K=\frac{\rho_{0} c^{2}}{\xi}$ is the bulk modulus that depends on the speed of sound $c . \xi$ is a dimensionless constant that is set at 7 for fluids to obtain a nearly incompressible fluid, and is set at 1 for compressible solids [27].

\subsection{Viscoelastic solid}

The stress tensor $\boldsymbol{\sigma}$ for an elastic solid consists of the hydrostatic and deviatoric stress:

$$
\sigma_{i}^{\alpha \beta}=-p_{i} \delta^{\alpha \beta}+S_{i}^{\alpha \beta}
$$

written in Einstein notation with respect to the coordinate indices $\alpha$ and $\beta$, with $\boldsymbol{\delta}$ denoting the Kronecker delta and $\boldsymbol{S}$ the deviatoric stress. In order to model a viscoelastic solid the deviatoric stress is added to the pressure term of the conservation of momentum in Eq. (5), in order to yield [20, 21]:

$$
F_{i}^{\sigma \alpha}=m_{i} \sum_{j} m_{j}\left(\frac{\sigma_{i}^{\alpha \beta}}{\rho_{i}^{2}}+\frac{\sigma_{j}^{\alpha \beta}}{\rho_{j}^{2}}\right) \nabla_{i}^{\beta} W_{i j} .
$$

The deviatoric stress according to Hooke's law reads:

$$
S^{\alpha \beta}=2 G\left(\epsilon^{\alpha \beta}-\frac{1}{3} \delta^{\alpha \beta} \epsilon^{\gamma \gamma}\right),
$$

with $G$ the shear modulus and $\epsilon$ the strain tensor. The Jaumann rate of change of the deviatoric stress is then calculated by:

$$
\frac{\mathrm{d} S_{i}^{\alpha \beta}}{\mathrm{d} t}=2 G\left(\dot{\epsilon}_{i}^{\alpha \beta}-\frac{1}{3} \delta^{\gamma \gamma} \dot{\epsilon}_{i}^{\alpha \beta}\right)+S_{i}^{\alpha \beta} \Omega_{i}^{\beta \gamma}+\Omega_{i}^{\alpha \gamma} S_{i}^{\gamma \beta}
$$


with $\dot{\boldsymbol{\epsilon}}$ the strain rate tensor:

$$
\dot{\epsilon}^{\alpha \beta}=\frac{1}{2}\left(\frac{\partial v^{\alpha}}{\partial x^{\beta}}+\frac{\partial v^{\beta}}{\partial x^{\alpha}}\right)
$$

and $\boldsymbol{\Omega}$ the spin tensor:

$$
\Omega^{\alpha \beta}=\frac{1}{2}\left(\frac{\partial v^{\alpha}}{\partial x^{\beta}}-\frac{\partial v^{\beta}}{\partial x^{\alpha}}\right)
$$

\subsection{Viscoelastic NSPH}

Processes at the cellular scale (i.e. $\mu$ m-scale) occur at a very low Reynolds number, meaning that the viscous forces dominate over the inertial forces leading to an overdamped system. Overdamped systems are generally assumed in agent based models of cells [19]. This means that the inertial forces in the conservation of momentum equation (4) can be omitted leading to the following equation for NSPH:

$$
-m_{i} \sum_{j} m_{j} \frac{\mu_{i}+\mu_{j}}{\rho_{i} \rho_{j}} \frac{\boldsymbol{r}_{i j} \cdot \nabla_{i} W_{i j}}{\left|\boldsymbol{r}_{i j}\right|^{2}+\eta^{2}} \boldsymbol{v}_{i j}=m_{i} \sum_{j} m_{j}\left(\frac{\boldsymbol{\sigma}_{i}}{\rho_{i}^{2}}+\frac{\boldsymbol{\sigma}_{j}}{\rho_{j}^{2}}\right) \cdot \nabla_{i} W_{i j}+\boldsymbol{F}_{i}^{b} .
$$

As Van Liedekerke et al. showed, we can rewrite the left hand side of this equation by introducing a friction matrix $\Gamma$ and by assuming that $m_{i}=m_{j}$ [18]:

$$
\sum_{j} \Gamma_{i j} \boldsymbol{v}_{i j}=\sum_{j}\left(\boldsymbol{\sigma}_{i} V_{i}^{2}+\boldsymbol{\sigma}_{j} V_{j}^{2}\right) \cdot \boldsymbol{\nabla}_{i} W_{i j}+\boldsymbol{F}_{i}^{b}
$$

with $V_{i}=\frac{m_{i}}{\rho_{i}}$ the particle volume and with the friction matrix:

$$
\Gamma_{i j}=\left(\mu_{i}+\mu_{j}\right) V_{i} V_{j} \frac{\boldsymbol{r}_{i j} \cdot \nabla_{i} W_{i j}}{\left|\boldsymbol{r}_{i j}\right|^{2}+\eta^{2}} .
$$

By solving this equation with a Conjugate Gradient Method 28, the velocities of the particles are obtained instead of accelerations. Van Liedekerke et al. demonstrated that NSPH is able to solve creeping flow problems with a time step of up to three orders of magnitude higher than SPH [18]. 


\subsection{Boundary Conditions}

Contact between different materials (e.g. cell and ECM) generates boundaries. Because the material properties of SPH are smoothed out, treating boundary conditions is one of the most challenging parts. Rigid boundaries of a material or a fixed wall are smoothed out and particles at the edge lack neighbors, leading to relatively high errors in the calculation of the density and deviatoric stress. Since accurate boundary conditions are important in many simulations, it is important to use an approach that minimizes these errors and provides the correct physical behavior. In a method proposed by Kulasegaram et al. 23] and later extended by Ferrand et al. 22. for the contact of a fluid with rigid boundaries, a boundary is represented by a single line of particles connected by line segments and wall boundary conditions are applied by renormalizing the conservation laws to correct for the missing part of the kernel volume behind the boundary. Here, this method is extended to allow to model contact between a moving rigid boundary and a viscous fluid or a viscoelastic solid modeled by $\mathrm{NSPH}$. A contact criterion is added to prevent overestimation of the hydrostatic pressure and deviatoric stress at the interface when a material is approaching a boundary.

\subsubsection{Renormalization factor}

For particles close to a rigid boundary which consists of a single line of particles, the assumption that the kernel support is completely filled with boundary particles does not hold (see Fig. 1). This leads to an incorrect estimation of property values for these particles. Kulasegaram et al. proposed to correct for the missing kernel volume by renormalizing the equation for the approximation of the density [23:

$$
\rho_{i}=\frac{1}{\gamma_{i}} \sum_{j \in M} m_{j} W_{i j},
$$


with $M$ the set of particles consisting of both the particles in the material as well as on the boundary and with $\gamma_{i}$ the renormalization factor:

$$
\gamma_{i}=\int_{\Omega \cap \Omega_{i}} W\left(\mathbf{r}^{\prime}-\mathbf{r}_{i}\right) d V^{\prime}
$$

with $\Omega$ the computational domain and $\Omega_{i}$ the kernel support. This means that $\gamma_{i}$ will have a value of 1 for particles inside a material and a value between 0 and 1 for particles at the boundary. In SPH it is not the density that is calculated in every time step, but the change in density with time. After introducing the renormalization factor, the conservation of mass, calculated using the product rule, becomes:

$$
\frac{\mathrm{d} \rho_{i}}{\mathrm{~d} t}=\frac{1}{\gamma_{i}} \sum_{j \in M} m_{j} \mathbf{v}_{i j} \cdot \nabla W_{i j}-\frac{\rho_{i}}{\gamma_{i}} \mathbf{v}_{i} \cdot \nabla \gamma_{i}
$$

assuming a static boundary and with the gradient of the renormalization factor:

$$
\boldsymbol{\nabla} \gamma_{i}=\int_{\Omega \cap \Omega_{i}} \boldsymbol{\nabla}_{i} W\left(\mathbf{r}^{\prime}-\mathbf{r}_{i}\right) d V^{\prime}=\int_{\partial \Omega \cap \Omega_{i}} W\left(\boldsymbol{r}^{\prime}-\boldsymbol{r}_{i}\right) \boldsymbol{n} d S^{\prime},
$$

where the surface integral is obtained using Gauss's theorem and with $\partial \Omega$ the boundary of $\Omega$ and $\boldsymbol{n}$ the inward normal at the boundary of the domain.

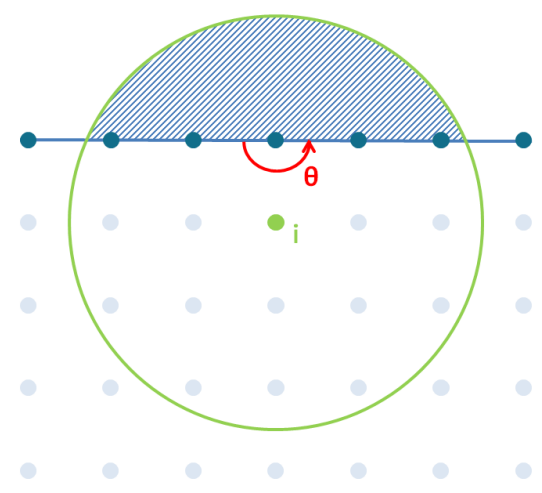

Figure 1: Illustration of the boundary situation in SPH. The boundary is represented by blue particles and line segments. For a material particle $i$ close to the boundary the kernel is not completely filled and information from the hatched area is missing. The initial volume of the boundary particles is calculated based on the angle $\theta$ between the connected line segments. 
As mentioned in [22], the initial volume of the boundary particles is defined by the angle between the two line segments connected to the particle (see Fig. 1). This means that the initial volume is half the volume of particles in the material for a boundary particle on a straight boundary and slightly larger for particles on a circle as will be seen later on.

2.5.2. Calculating the renormalization factor and gradient of the renormalization factor

Rigid boundaries are modeled as a single line of particles (called boundary particles $b$ ), connected with lines which will be called segments $z$. For a material particle, $\gamma$ can now be obtained by summing over and calculating the kernel volume behind these segments. An illustration of this process for one segment is shown in Fig. 2 The renormalization factor is calculated as:

$$
\gamma_{i}=1-\sum_{z \in Z} \cos (\alpha) l_{z} \int_{r_{q}}^{2 h} \frac{r}{r_{q}} W(r) d r .
$$

A quadrature rule is used to accurately calculate the kernel volume behind the boundary. For each quadrature point $q$ on line segment $z$ (in the set $Z$ containing all line segments) the integral is calculated analytically, with $r$ the distance to particle $i, r_{q}$ the distance between the quadrature point and the particle, $l_{z}$ the length of the line segment and $\alpha$ the angle between the line from the particle to the quadrature point and the normal vector to $z$ (see Fig. 22). 


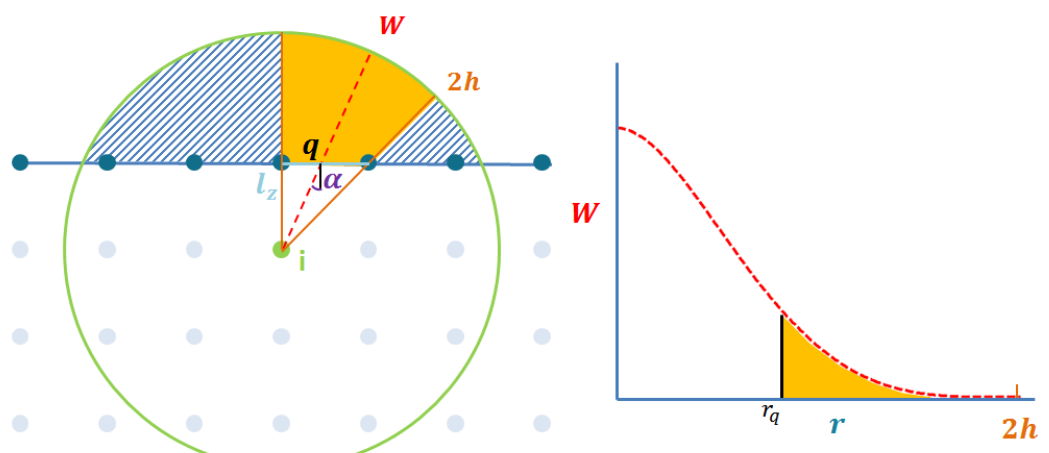

Figure 2: Renormalization factor calculation. Left: Top view. The kernel volume behind each boundary segment $z$ for a material particle $i$ close to a boundary (dark dots and segments) is calculated and summed to obtain the total missing kernel volume behind the boundary. Right: Side view. Kernel value as function of the distance $r$ to the particle. The missing volume behind the boundary segment $z$ is shown in orange.

The gradient of the renormalization factor is calculated as:

$$
\nabla \gamma_{i}=\sum_{z \in Z} \int_{z} W(r) \boldsymbol{n} d S
$$

with the gradient of the renormalization factor for one specific segment as:

$$
\nabla \gamma_{i z}=\left(\int_{b 1}^{b 2} W(r) d l\right) \boldsymbol{n}_{z}
$$

with $b_{1}$ and $b_{2}$ the boundary particles connected by the segment $z$ and $\boldsymbol{n}_{z}$ the inward normal vector of segment $z$.

\subsubsection{Renormalization of conservation laws}

In Eq. 19, the adapted equation for the conservation of mass is shown in the case of a rigid boundary. If the boundary is also allowed to move, the conservation of mass becomes:

$$
\frac{\mathrm{d} \rho_{i}}{\mathrm{~d} t}=\frac{1}{\gamma_{i}} \sum_{j \in M} m_{j} \boldsymbol{v}_{i j} \cdot \nabla W_{i j}-\frac{\rho_{i}}{\gamma_{i}} \sum_{z \in Z} \mathbf{v}_{i z} \cdot \nabla \gamma_{i z}
$$


The equation of momentum is adapted in the same way as in [22, but now with the full stress tensor $\boldsymbol{\sigma}$ instead of only the hydrostatic pressure:

$$
\boldsymbol{F}_{i}^{\sigma}=\frac{m_{i}}{\gamma_{i}} \sum_{j \in M} m_{j}\left(\frac{\boldsymbol{\sigma}_{i}}{\rho_{i}^{2}}+\frac{\boldsymbol{\sigma}_{j}}{\rho_{j}^{2}}\right) \cdot \nabla W_{i j}+\frac{m_{i} \rho_{i}}{\gamma_{i}} \sum_{z \in Z}\left(\frac{\boldsymbol{\sigma}_{i}}{\rho_{i}^{2}}+\frac{\boldsymbol{\sigma}_{z}}{\rho_{z}^{2}}\right) \rho_{z} \cdot \nabla \gamma_{i z}
$$

The rate of change of the deviatoric stress is also renormalized and becomes:

$\frac{\mathrm{d} S_{i}^{\alpha \beta}}{\mathrm{d} t}=\frac{1}{\gamma_{i}} 2 G\left(\dot{\epsilon}_{i}^{\alpha \beta}-\frac{1}{3} \delta^{\gamma \gamma} \dot{\epsilon}_{i}^{\alpha \beta}\right)+\frac{1}{\gamma_{i}} S_{i}^{\alpha \beta} \Omega_{i}^{\beta \gamma}+\frac{1}{\gamma_{i}} \Omega_{i}^{\alpha \gamma} S_{i}^{\gamma \beta}-\frac{S_{i}^{\alpha \beta}}{\gamma_{i}} \sum_{z \in Z} \mathbf{v}_{i z} \cdot \nabla \gamma_{i z}$.

The term for the viscous forces according to Morris et al. [26] in the conservation of momentum is adapted as in [29]:

$$
\boldsymbol{F}_{i}^{v}=\frac{m_{i}}{\gamma_{i}} \sum_{j \in M} m_{j} \frac{\mu_{i}+\mu_{j}}{\rho_{i} \rho_{j}} \frac{\boldsymbol{r}_{i j} \cdot \nabla_{i} W_{i j}}{\left|\boldsymbol{r}_{i j}\right|^{2}+\eta^{2}} \boldsymbol{v}_{i j}-\frac{m_{i}}{\gamma_{i} \rho_{i}} \sum_{z \in Z} \frac{\boldsymbol{v}_{i z} \cdot \boldsymbol{t}_{i z}}{\delta r_{i z}} \boldsymbol{t}_{i z}\left|\nabla \gamma_{i z}\right|
$$

with $\boldsymbol{t}_{i z}$ a unit vector along the tangential component of the velocity of particle $i$ with respect to segment $z$ :

$$
\boldsymbol{t}_{i z}=\frac{\boldsymbol{v}_{i z}-\left(\boldsymbol{v}_{i z} \cdot \boldsymbol{n}_{z}\right) \cdot \boldsymbol{n}_{z}}{\left|\boldsymbol{v}_{i z}-\left(\boldsymbol{v}_{i z} \cdot \boldsymbol{n}_{z}\right) \cdot \boldsymbol{n}_{z}\right|}
$$

and

$$
\delta r_{i z}=\max \left(\boldsymbol{r}_{i z} \cdot \boldsymbol{n}_{z}, d p\right)
$$

with $d p$ the interparticle distance.

\subsubsection{Setting properties for boundary particles and segments}

In order to solve the equations in the sections above, the properties at the boundary particles and boundary segments need to be computed. As was shown by Ferrand et al. an SPH interpolation is used to calculate the density and hydrostatic pressure for the boundary particles assuming the boundary condition 
$\frac{\partial \rho}{\partial n}=0$ with $\boldsymbol{n}$ the normal to the boundary [22]:

$$
\rho_{b}=\frac{\sum_{j \in M \backslash B} m_{j} W_{j b}}{\sum_{j \in M \backslash B} \frac{m_{j}}{\rho_{j}} W_{j b}},
$$

with $B$ the set of all SPH boundary particles and

$$
p_{b}=\rho_{b} \frac{\sum_{j \in M \backslash B} \frac{m_{j}}{\rho_{j}}\left(\frac{p_{j}}{\rho_{j}}-\boldsymbol{a}_{j} \cdot \boldsymbol{r}_{j b}+\frac{1}{2}\left(\boldsymbol{v}_{j}-\boldsymbol{v}_{b}\right) \cdot \boldsymbol{r}_{j b}\right) W_{j b}}{\sum_{j \in M \backslash B} \frac{m_{j}}{\rho_{j}} W_{j b}},
$$

with $\boldsymbol{a}_{j}$ the acceleration caused by the body forces $\boldsymbol{F}_{j}$. The deviatoric stress tensor for the boundary particles is calculated similarly:

$$
\boldsymbol{S}_{b}=\rho_{b} \frac{\sum_{j \in M \backslash B} \frac{m_{j}}{\rho_{j}} \frac{\boldsymbol{S}_{j}}{\rho_{j}} W_{j b}}{\sum_{j \in M \backslash B} \frac{m_{j}}{\rho_{j}} W_{j b}} .
$$

The same properties are calculated for the boundary segments by taking the average property values of the two boundary particles belonging to the respective boundary segment.

\subsubsection{Contact criterion}

Since we are defining a discrete boundary in the smoothed SPH method, errors in both density and deviatoric stress calculation are being made when a material approaches a boundary. In order to ensure a correct estimation of the hydrostatic pressure and deviatoric stress tensor when a material approaches a boundary, a contact criterion is introduced as illustrated in Fig. 3 . With the introduced boundary renormalization method a material starts to build up pressure and deviatoric stress as soon as it is within a distance equal to the kernel width (generally chosen between 2 and 4 times the interparticle distance) from the boundary. However, the pressure and deviatoric stress inside a material are zero when all particles are at exactly one (preset) interparticle distance away from their closest neighbors. Therefore, the pressure and deviatoric stress 
should only start to build up when the material is within a distance equal to the same interparticle distance from the boundary. We propose to add a contact criterion that aims to reduce the error related to premature stress buildup. This contact criterion discretizes the material-boundary interface which is normally smoothed in SPH.

Contact between the material and the boundary is established when material particles are within one interparticle distance of the boundary. When a particle is in contact with the boundary, the interaction between the particle and the boundary will be added to the conservation equations. From the moment that a material particle is in contact with the boundary, hydrostatic pressure and deviatoric stress should be built up not only in this particle, but also in the material particles surrounding the particle in contact. However, particles at the surface of the material next to the particle in contact that are not yet within one interparticle distance from the boundary should not be considered to be in contact with the boundary yet. Therefore, the contact criterion consist of two steps. In the first step all particles that are within one interparticle distance from the boundary are considered in contact and will be called "primary contact particles" hereafter. In the second step all particles within the kernel support of the primary contact particles that are not located at the surface of the material are also considered in contact with the boundary and are called "secondary contact particles". In order to distinguish the secondary contact particles from particles on the surface of the material a kernel support value is calculated for each particle:

$$
\psi_{i}=\sum_{j \in M \backslash B} \frac{m_{j}}{\rho_{j}} W_{i j}
$$

which will be approximately 1 for particles in a material and lower for particles close to the surface of a material. A threshold value (here chosen to be 0.8 ) is chosen above which particles are to be selected as secondary contact particles. An illustration of the contact criterion is shown in Fig. 3. 


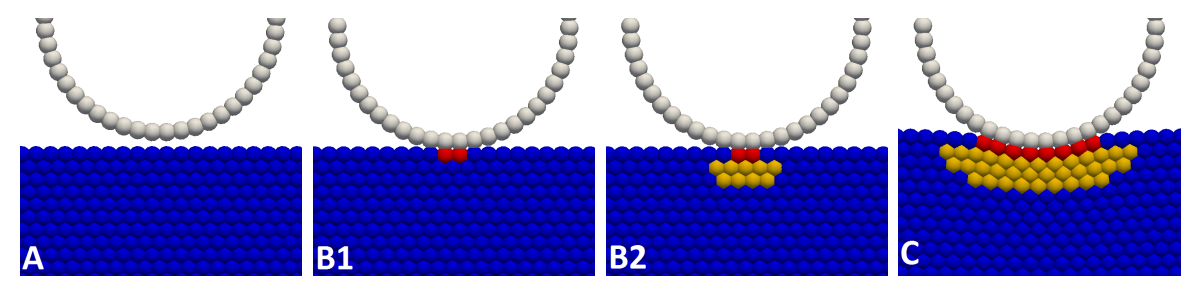

Figure 3: Various steps in defining contact using the contact criterion for a material in contact with a circular indenter. (A) The distance between the material and the indenter is more than one interparticle distance, thus no contact is established. (B1) The indenter comes within one interparticle distance from 2 material particle. These particles are selected as "primary contact particles" (shown in red). (B2) In a second step all particles within the kernel support of a primary contact particle that are not located at the surface of the material are selected as "secondary contact particles" (shown in yellow). (C) When the material is indented more material particles come in contact with the indenter.

Since the total force that acts on a particle changes when it establishes contact with the boundary, during simulations a particle may establish and lose contact regularly. This means that a particle can move towards the boundary when it is not in contact and move away from the boundary when it is in contact. If in this fluctuating state of establishing and losing contact a particle moves away from the boundary, the hydrostatic pressure of the particle will decrease. However, if a particle that has lost contact moves back towards the boundary, the hydrostatic pressure will not increase because no contact is established with the boundary. Therefore, the hydrostatic pressure of the particle starts to decrease without a net displacement of the particle relative to the boundary. As this change in hydrostatic pressure (and deviatoric stress) is obviously not physical, an additional rule is added to the contact criterion to prevent this. Primary contact particles can only lose contact if they are at a distance from the boundary which is slightly larger than the interparticle distance. In this way the stability of the simulations is improved, without affecting too much the accuracy of the hydrostatic pressure and deviatoric stress calculation. 


\subsection{Time stepping}

In every time step for NSPH properties are calculated in the order as indicated in Fig. 4. $\rho, \boldsymbol{S}$ and particle position $\boldsymbol{x}$ are integrated using the explicit Euler method.

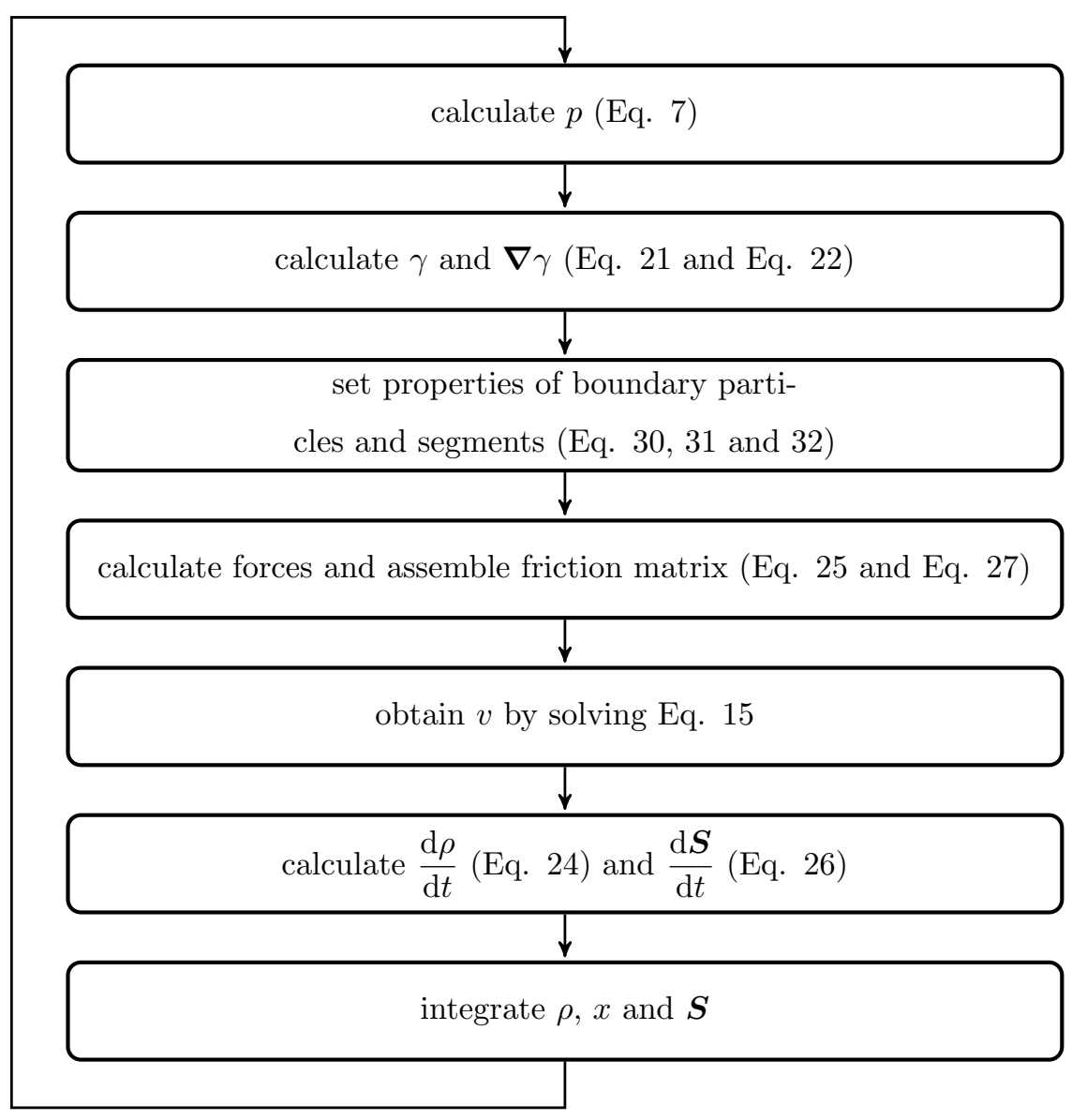

Figure 4: Diagram of all steps taken in a single time step in an (N)SPH simulation.

\section{Results}

In order to demonstrate the effectiveness and accuracy of the viscoelastic NSPH method in combination with the extended boundary conditions, four test simulations are performed: tractions applied to a viscoelastic solid, Stokes 
flow around an array of rigid square pillars, indentation with a rigid, yet moving, circular indenter of a viscoelastic solid and cell-ECM interaction with cell migration through a viscoelastic ECM by local degradation.

In [18 it was demonstrated that for $\mathrm{SPH}$ the maximal time step $\Delta t$ is a quadratic function of the kernel width:

$$
\Delta t \leq 0.125 \frac{h^{2} \rho}{\mu}
$$

with $h$ the smoothing length, $\rho$ the density and $\mu$ the dynamic viscosity of the material. At the $\mu \mathrm{m}$-scale, a huge number of calculation steps would be required to even simulate a process at the timescale of seconds. Therefore, although cell dynamics takes place at the $\mu \mathrm{m}$-scale, for comparison between NSPH and SPH simulations have been performed at a large length scale. For the simplified model of cell migration in Section 3.4 only NSPH is used and simulations are performed at $\mu \mathrm{m}$-scale. The cubic spline kernel is used in simulations unless stated otherwise. All simulations are performed using the $\mathrm{C}++$ particle-based software called Mpacts (http://dem-research-group.com).

\subsection{Deformation of a viscoelastic solid by tractions}

In a $2 \mathrm{D}$ square shaped viscoelastic solid (Young's modulus $\mathrm{E}=2.5 \mathrm{~Pa}$, Poisson's ratio $\nu=0.45$ from which the bulk modulus $K=\frac{E}{3(1-2 \nu)}$ and shear modulus $G=\frac{E}{2(1+\nu)}$ are calculated, dynamic viscosity $\mu=100 \mathrm{~Pa} \cdot \mathrm{s}$ and initial density $\rho_{0}=1000 \mathrm{~kg} / \mathrm{m}^{3}$ ) with side length $\mathrm{L}=2 \mathrm{~m}$ and particle spacing of $2.5 \times 10^{-2} \mathrm{~m}$ on a cubic lattice (smoothing length $\mathrm{h}=3.25 \times 10^{-2} \mathrm{~m}$ ) three circular regions of radius $0.2 \mathrm{~m}$ are selected. A body force of $6.25 \times 10^{-4} \mathrm{~N}$ is applied instantaneously to particles within these circles in a direction indicated in Fig. 5 to create a displacement field in the material. The square domain is surrounded by stationary particles that function as a rigid boundary. Simulations have been run until an end time of $1000 \mathrm{~s}$ for both SPH and NSPH, with a time step of $2.5 \times 10^{-4} \mathrm{~s}$ for $\mathrm{SPH}$ and $10 \mathrm{~s}$ for NSPH simulations. The material displaces with a maximal velocity of $5.0 \times 10^{-4} \mathrm{~m} / \mathrm{s}$ and hence the Reynolds number equals 
$\operatorname{Re}=0.01\left(\operatorname{Re}=\frac{\rho v L}{\mu}\right.$, with the initial density $\rho=1000 \mathrm{~kg} / \mathrm{m}^{3}$, the maximal velocity $v=5.0 \times 10^{-4} \mathrm{~m} / \mathrm{s}$, the characteristic length scale $L=2 \mathrm{~m}$ and the dynamic viscosity $\mu=100 \mathrm{~Pa} \cdot \mathrm{s})$.

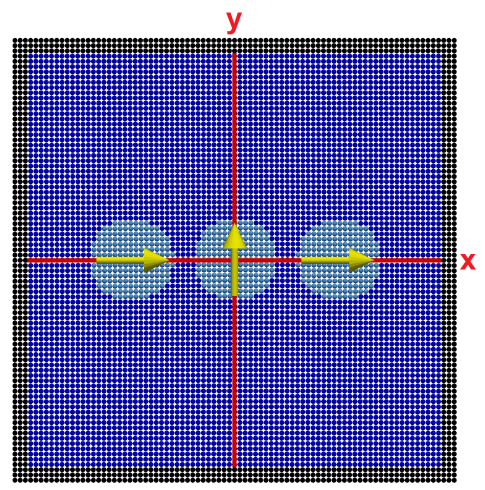

Figure 5: Particle representation of a viscoelastic solid with selected circular patches to which body forces are applied in the indicated direction. Displacement profiles shown in Fig. 7 are taken from particles on the horizontal $(\mathrm{x})$ and vertical $(\mathrm{y})$ line.

A comparison is made between $\mathrm{SPH}, \mathrm{NSPH}$ and a FE simulation serving as a reference for both the equilibrium (i.e. elastic) solution as well as the transient response. For the FE simulation Abaqus V6.14 is used with biquadratic plane strain quadrilateral elements with a grid size fine enough to ensure convergence (element edge length ranging from $0.015 \mathrm{~m}$ to $0.067 \mathrm{~m}$ ). The viscoelastic solid is simulated as a Kelvin-Voigt material for which the Prony series parameters are obtained as explained in Appendix A

Displacement fields at equilibrium for FE, SPH and NSPH are shown in Fig. 6. Although viscoelastic NSPH requires only 100 time steps, the resulting displacement is equal to that obtained with viscoelastic SPH and agrees very well with the FE simulation with a relative error of $0.38 \%$ for the peak displacement. Next, displacement profiles are taken at different time points in the SPH, NSPH and FE simulation along the $\mathrm{x}$ - and $\mathrm{y}$-axis (see Fig. 5). Displacement profiles at equilibrium (1000s) show very good resemblance between SPH, NSPH and FE. Moreover, the SPH, NSPH and FE profiles evolve similarly in time, with 
only small differences at the first time step (after $10 \mathrm{~s}$ ). This demonstrates that viscoelastic NSPH can capture the viscoelastic behavior of a solid with the same accuracy as both SPH and FE.

In order to demonstrate that viscoelastic NSPH can also be used to model the ECM, a comparison is made between NSPH and FE for a simulation at the $\mu \mathrm{m}$-scale $(\mathrm{L}=200 \mu \mathrm{m})$ and with a more realistic ECM Young's modulus $(\mathrm{E}=2.5 \mathrm{kPa})$, yielding a more representative application of cellular tractions to the ECM through focal adhesions. The transient displacement profiles obtained for these simulations (not shown here) are exactly the same, but scaled with respect to the results in Figure 7 indicating that viscoelastic NSPH can capture the mechanical behavior of a viscoelastic solid that represents the ECM at relevant length scales.
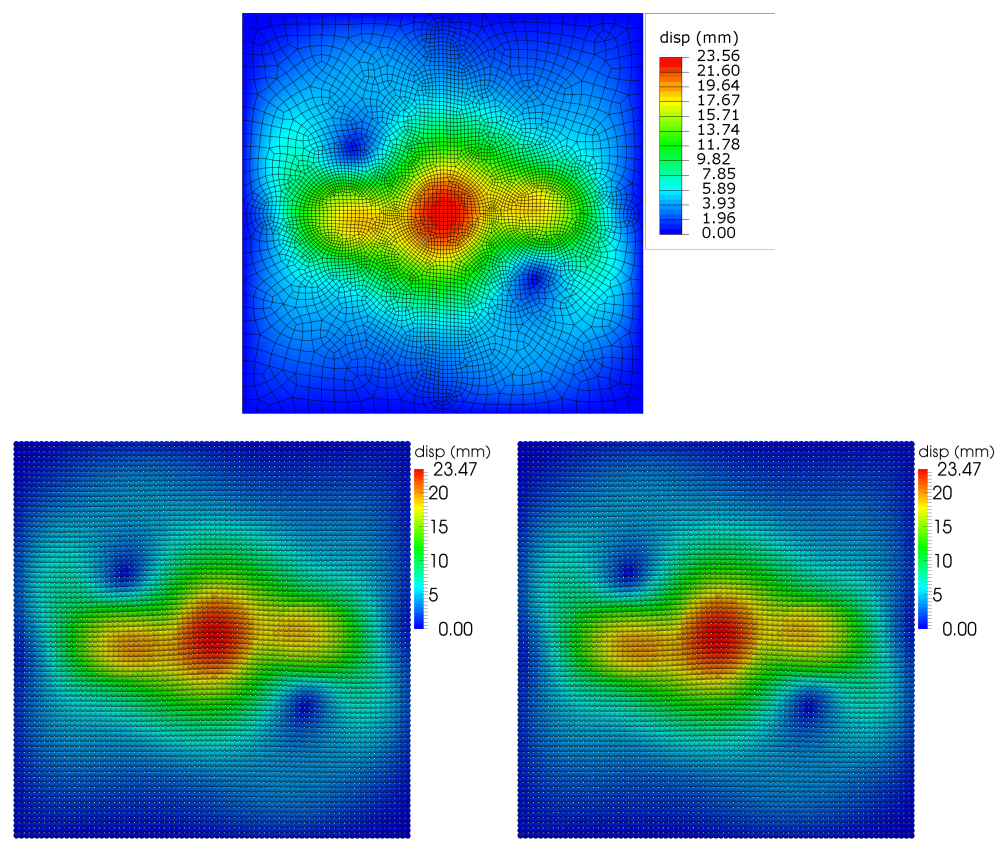

Figure 6: Total displacement magnitudes at equilibrium for circular patches of body forces (see Fig. 5 calculated by means of FE (top), SPH (bottom left) and NSPH (bottom right). 

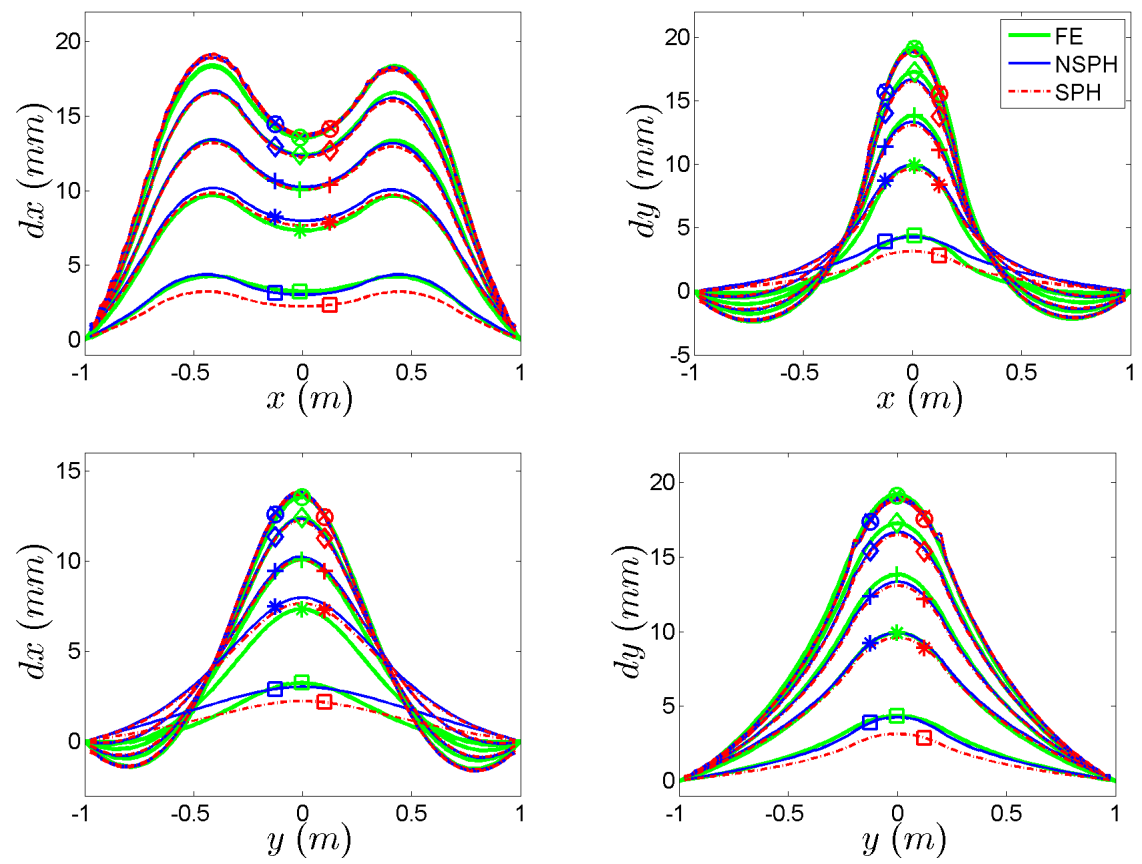

Figure 7: Displacement profiles for circular patches of body forces taken from particles on the horizontal and vertical line (see Fig. 5 at different time points for FE, SPH and NSPH. Profiles show either the displacement of particles in $\mathrm{x}$-direction $(\mathrm{dx})$ or in $\mathrm{y}$-direction $(\mathrm{dy})$. Profiles are obtained after $10(\square), 50(*), 100(+), 200(\diamond), 500(\circ)$ and $1000 \mathrm{~s}(\times)$ of the simulations, with $1000 \mathrm{~s}$ being equal to $4 \times 10^{6}$ steps for SPH and 100 steps for NSPH.

\subsection{Fluid flow around an array of square pillars}

Fluid flow around an array of square pillars is simulated by placing a rigid, stationary 2D square pillar with a side length of $4.0 \times 10^{-3} \mathrm{~m}$ in a $2 \mathrm{D}$ square fluid domain $\left(\mu=5 \times 10^{-2} \mathrm{~Pa} \cdot \mathrm{s}, \mathrm{K}=2 \mathrm{~Pa}\right.$ and $\left.\rho_{0}=1000 \mathrm{~kg} / \mathrm{m}^{3}\right)$ domain with side length $10^{-2} \mathrm{~m}$. In y-direction stationary particles are used as a rigid boundary. In x-direction periodic boundaries are imposed and implemented using ghost particles, leading to the simulation of fluid flow around an array of square pillars. 


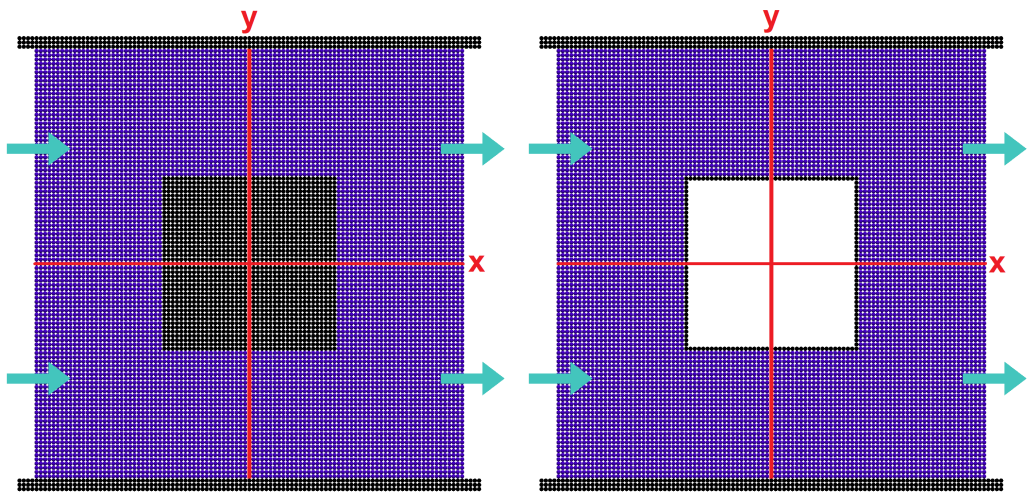

Figure 8: Particle system of flow around an array of square pillars either filled up completely with rigid particles (left) or represented by the extended boundary method (right). The fluid (blue) is constrained in y-direction by non moving boundary particles and in x-direction periodic boundary conditions are applied. Velocity profiles are obtained for particles initially located on the vertical (y) red line.

A particle spacing of $1 \times 10^{-4} \mathrm{~m}$ on a cubic lattice $\left(\mathrm{h}=1.3 \times 10^{-4} \mathrm{~m}\right)$ is used and a body force of $2.2 \mathrm{nN}$ is applied to all fluid particles, resulting in a steady state velocity distribution in the fluid with $R e=2 \times 10^{-4}$. Simulations have been performed with the square pillar being either filled up completely with rigid particles or represented by the extended boundary method for both SPH and NSPH. The contact criterion is not used here since the fluid is already in contact with the boundary. A time step of $5 \times 10^{-5} \mathrm{~s}$ for SPH and $2 \times 10^{-3} \mathrm{~s}$ for NSPH simulations is used. The velocity profiles along the y-axis (see Fig. 8) are shown in Fig. 9 at various time steps for NSPH and at steady state for $\mathrm{SPH}$. For NSPH, as was observed for a similar simulation in [18, a steady state flow is reached within 10 time steps and is in close agreement with the steady state solution obtained for SPH after 10000 time steps. The relative difference in peak velocity between NSPH after 20 time steps and SPH after 10000 time steps in the simulation with extended boundary method is $0.30 \%$. Further, the velocity profiles for both boundary methods for SPH as well as NSPH agree very well, demonstrating that coupling of NSPH with the extended boundary 
method works well for the interaction of a rigid, stationary boundary with a fluid.
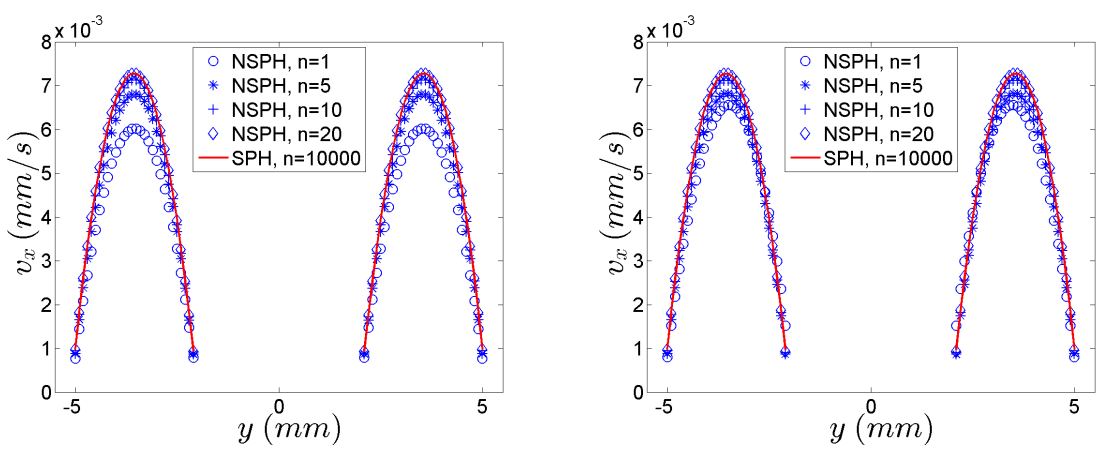

Figure 9: Velocity profiles for flow around an array of square pillars. Fluid velocity in $\mathrm{x}-$ direction for particles initially on the y-axis (see Fig. 8 is shown for SPH as well as NSPH for a pillar filled with rigid particles (left) and a pillar represented by the extended boundary method (right). Profiles are shown at different steps $n$ for NSPH and at steady state $(n=10000)$ for SPH.

\subsection{Indentation of a viscoelastic solid with a circular indenter}

Indentation of a viscoelastic solid with a rigid, yet moving indenter (radius $0.4 \mathrm{~m}$ ) is simulated by indenting a $2 \mathrm{D}$ square shaped viscoelastic solid $\left(\mathrm{E}=2.5 \mathrm{~Pa}, \nu=0.45, \mu=100 \mathrm{~Pa} \cdot \mathrm{s}\right.$ and $\left.\rho_{0}=1000 \mathrm{~kg} / \mathrm{m}^{3}\right)$ with a side length $\mathrm{L}=1$ $\mathrm{m}$ and particle spacing of $2.5 \times 10^{-2} \mathrm{~m}$ on a hexagonal lattice $\left(\mathrm{h}=3.25 \times 10^{-2} \mathrm{~m}\right)$. The square domain is supported at the bottom by stationary particles that serve as a rigid boundary. The indenter is modeled with the extended boundary method and is considered as a rigid body that can translate, but not rotate or deform. A force of $0.2 \mathrm{~N}$ is applied to the indenter and simulations are run for $2000 \mathrm{~s}$ with $\mathrm{Re}=0.01$. For a reference elastic FE simulation, the grid size is taken equal to the interparticle distance of the $(\mathrm{N}) \mathrm{SPH}$ simulations and a no slip condition is used for the contact between indenter and material. 

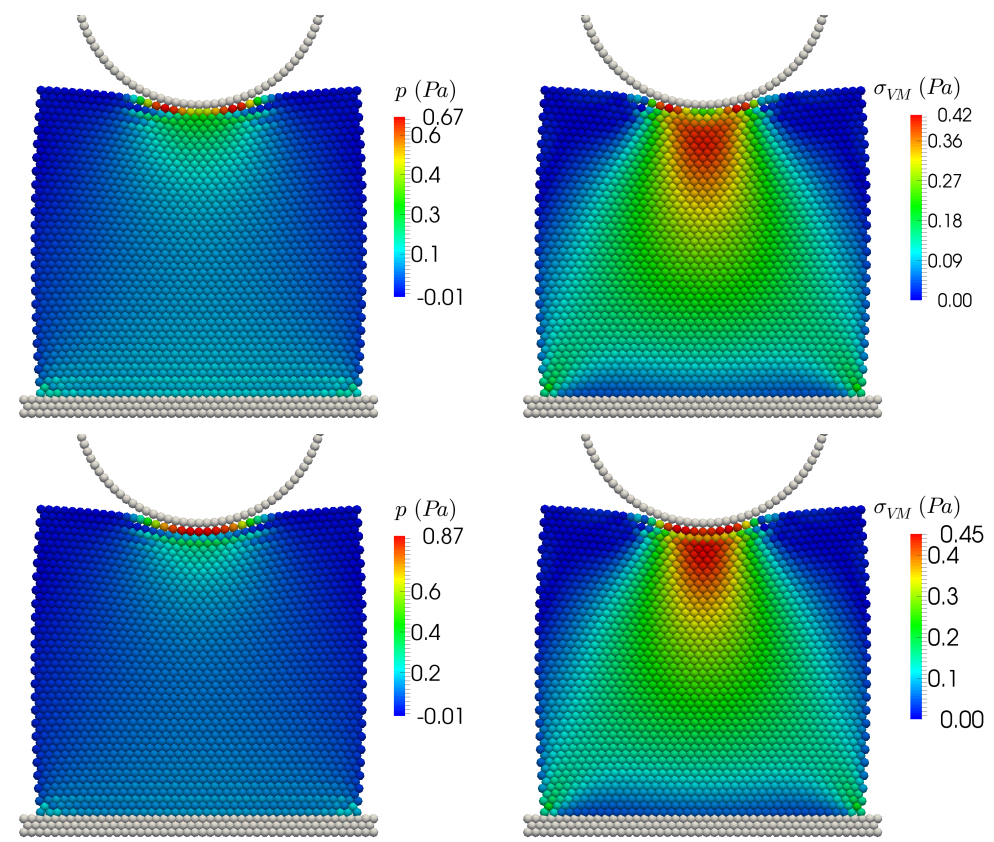

Figure 10: Hydrostatic pressure $p$ (left) and von Mises stress distribution $\boldsymbol{\sigma}_{\mathrm{VM}}$ (right) at equilibrium for indentation of a viscoelastic solid with a circular indenter modeled by means of NSPH $(\mathrm{Re}=0.01)$ without the use of a contact criterion. The indenter is initially positioned at a distance from the material equal to either one (top) or three (bottom) times the interparticle distance.

First, two NSPH simulations are performed without use of the contact criterion described in Section 2.5.5. The results of these simulations are shown in Fig. 10 for an indenter initially positioned at a distance from the material equal to either one or three times the interparticle distance. For both simulations spurious effects arise that are not observed for FE (see Fig. 11). For the indenter initially positioned at one interparticle distance from the material, maximum hydrostatic pressure and deviatoric stress are found at particles lateral from instead of on the vertical center line. This is caused by the fact that for these particles lateral from the center line the indenter is initially positioned at a larger distance above the material. Therefore, the indenter has to cover a larger distance relative to the material before contact is established with these 
particles compared to particles located on the center line. This larger relative displacement between the indenter and the material particles leads to an overestimation of the hydrostatic pressure and deviatoric stress for particles lateral from the center line. For the indenter initially positioned at three times the interparticle distance, the indenter has to cover a larger distance in order to establish contact with the material for all material particles. This leads to an overestimated hydrostatic pressure for the first line of particles in contact with the indenter. As a result of this overestimation a striated pattern of lower and higher hydrostatic pressure can be observed for layers of particles below the top layer. The same effect can be seen for the von Mises stress. 


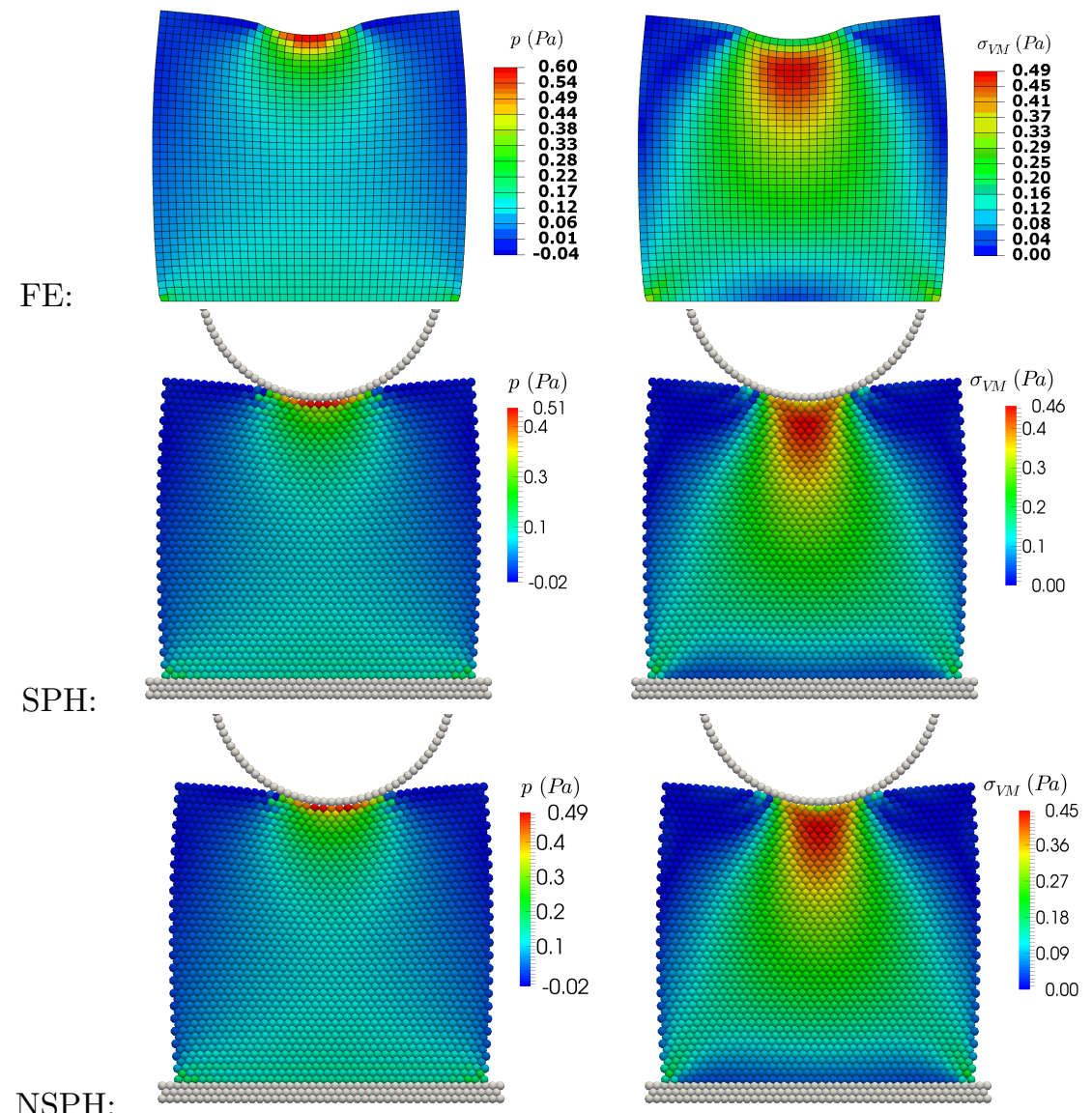

Figure 11: Hydrostatic pressure $p$ in $\mathrm{Pa}$ (left) and von Mises stress distribution $\boldsymbol{\sigma}_{\mathrm{VM}}$ in $\mathrm{Pa}$ (right) at steady state for indentation of a circular indenter in a viscoelastic solid modeled by means of FE (top), SPH (middle) and NSPH (bottom) $(\mathrm{Re}=0.01)$.

Next, SPH and NSPH simulations are performed with the contact criterion included. The distance from the boundary at which particles lose contact is set at 1.1 times the interparticle distance. The hydrostatic pressure and von Mises stress distribution at equilibrium are shown in Fig. 11. Hydrostatic pressure and von Mises stress distributions, obtained by means of SPH and NSPH, are comparable to those obtained by means of FE. The maximum von Mises stress is not located at the top surface, but slightly below. This is the consequence of the 
no-slip condition between the top surface and the indenter. The same effect can be observed for both the SPH and NSPH simulation, although the maximum von Mises stress value is slightly lower compared to FE. The maximum hydrostatic pressure value is lower as well, with relative errors of $15 \%$ and $18.3 \%$ respectively for SPH and NSPH, compared to FE. More importantly, the use of a proper boundary criterion leads to a smoother hydrostatic pressure and von Mises stress distribution and avoids the occurrence of spurious pressures and stresses when no contact criterion is being used. The hydrostatic pressure and von Mises stress profiles along the central vertical axis are shown in Fig. 12. It can be seen that SPH and NSPH simulations lead to profiles that are similar to those of FE, with some differences close to the boundary. Together, these results demonstrate that the extended boundary method clearly improves modeling of the contact between a viscoelastic solid and a moving boundary for both SPH and NSPH.

Similar to Section 3.1, a comparison is made between NSPH and FE for a simulation at the $\mu \mathrm{m}$-scale $(\mathrm{L}=100 \mu \mathrm{m})$ and with a more realistic ECM Young's modulus $(\mathrm{E}=2.5 \mathrm{kPa})$. Again, the hydrostatic pressure and von Mises stress profiles obtained by these simulations (not shown here) are equal, but scaled to the results of high length-scale simulations (see Figure 11), indicating that viscoelastic NSPH together with the extended boundary method can capture the mechanical behavior of a viscoelastic solid representing the ECM. 

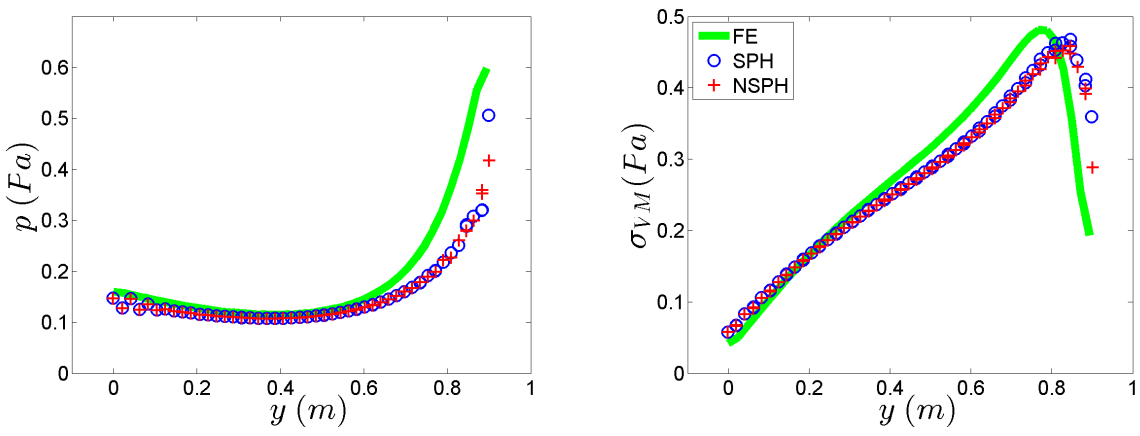

Figure 12: Hydrostatic pressure $p$ (left) and von Mises stress profile $\boldsymbol{\sigma}_{\mathrm{VM}}$ (right) along the central vertical axis at equilibrium for indentation of a viscoelastic solid modeled by means of FE, SPH and NSPH (Re=0.01).

In order to demonstrate that the extended boundary method is also valid for underdamped systems, an impact simulation is performed, modeled by means of SPH. An initial velocity of $0.2 \mathrm{~ms}^{-1}$ (instead of a force) is given to a rigid circular indenter with a mass of $3000 \mathrm{~kg}$. A 2D square shaped viscoelastic solid $\left(\mathrm{E}=2.5 \mathrm{kPa}, \nu=0.45, \mu=100 \mathrm{~Pa} \cdot \mathrm{s}\right.$ and $\left.\rho_{0}=1000 \mathrm{~kg} / \mathrm{m}^{3}\right)$ is indented, followed by indenter recoil and underdamped oscillatory deformations of the solid. The simulation is run for $50 \mathrm{~s}$. A time sequence is shown in Figure 13 and a supporting video of this simulation is attached to the online manuscript. It can be seen that, after oscillations have decreased to zero, the solid adopts almost to its initial square shape with small residual von Mises stress values $(<8 \%)$ compared to values during indentation. 


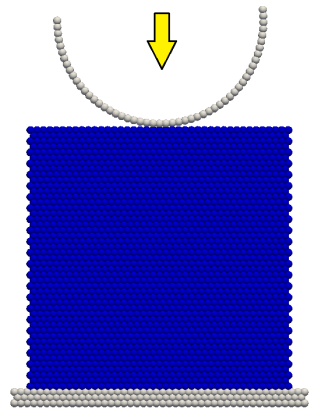

$0 \mathrm{~s}$

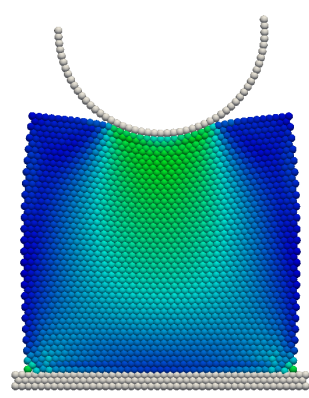

$3 \mathrm{~s}$

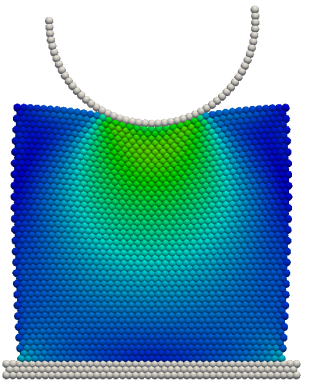

$0.6 \mathrm{~s}$

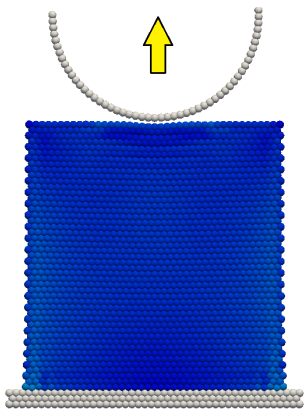

$3.8 \mathrm{~s}$

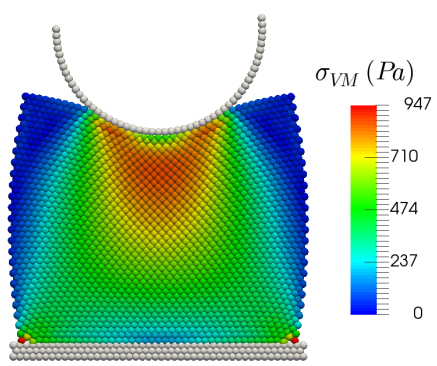

$1.8 \mathrm{~s}$

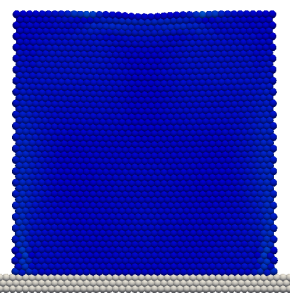

$50 \mathrm{~s}$

Figure 13: Von Mises stress distribution $\boldsymbol{\sigma}_{\mathrm{VM}}$ for various time steps during indentation and recoil of a viscoelastic solid by a circular indenter, modeled by means of SPH.

\subsection{Degradation-mediated cell migration through a viscoelastic ECM}

Having validated the implementations of $(\mathrm{N}) \mathrm{SPH}$ and the extended boundary method, simulations of cell migration in a deformable and degradable ECM are performed in order to highlight the advantages of using a viscoelastic NSPH description for the ECM. The cell is modeled as a rigid, circular self-propelling object similar to the indenter in Section 3.3 and embedded in a viscoelastic solid that can be locally degraded by the cell. The cell model used here is a strong simplification of a real cell. At the same time cells in agent-based models are often and successfully represented as simple geometrical objects (e.g. 30, 31]). ECM degradation is modeled as fluidization of the material, meaning that the 
deviatoric stress is relaxed while the hydrostatic pressure is not affected by the degradation. In a hydrogel this cell-mediated degradation would represent the cleavage of polymers by enzymes (proteinases like matrix metalloproteinases (MMPs)) secreted by the cell. By this cleavage, the connectivity of the polymer network is reduced which allows the cell to move through. Degradation is implemented by introducing a degradation factor $f_{\text {degr }}$ which is 1 for an intact viscoelastic solid and 0 for a completely degraded material. $f_{\text {degr }}$ is updated for each NSPH particle based on its distance to the cell center by solving the following differential equation:

$$
\frac{\mathrm{d} f_{\text {degr }, i}}{\mathrm{~d} t}=-\frac{1}{\tau_{\text {degr }}} \exp \left(-\frac{d_{i, \text { center }}-r_{\text {cell }}}{0.25 r_{\text {cell }}}\right)
$$

with $\frac{1}{\tau_{\text {degr }}}$ is a reference degradation rate that determines the degradation speed. $d_{i, \text { center }}$ is the distance of particle $i$ to the cell center and $r_{\text {cell }}$ is the radius of the cell. A minimal value of 0 for $f_{\text {degr }}$ is enforced in order to prevent negative values. In this way, particles closest to the cell undergo faster degradation than particles further away. Besides, degradation is limited to particles in front of the cell that are within a certain distance from the cell and within a certain angle (given by $\theta_{\text {degr }}$, see Fig. 14) with respect to the prescribed migration direction. It must be stated that the equation for degradation is arbitrarily chosen and is a simplification of the mechanics that govern degradation kinetics such as proteinase expression, section, transport and binding.

The degradation factor and its change in the current time step are used to adapt the NSPH conservation equations. The deviatoric stress tensor of each particle is relaxed:

$$
\frac{\mathrm{d} \boldsymbol{S}_{i}}{\mathrm{~d} t}=-\frac{\mathrm{d} f_{\mathrm{degr}}}{\mathrm{d} t} \boldsymbol{S}_{i}
$$



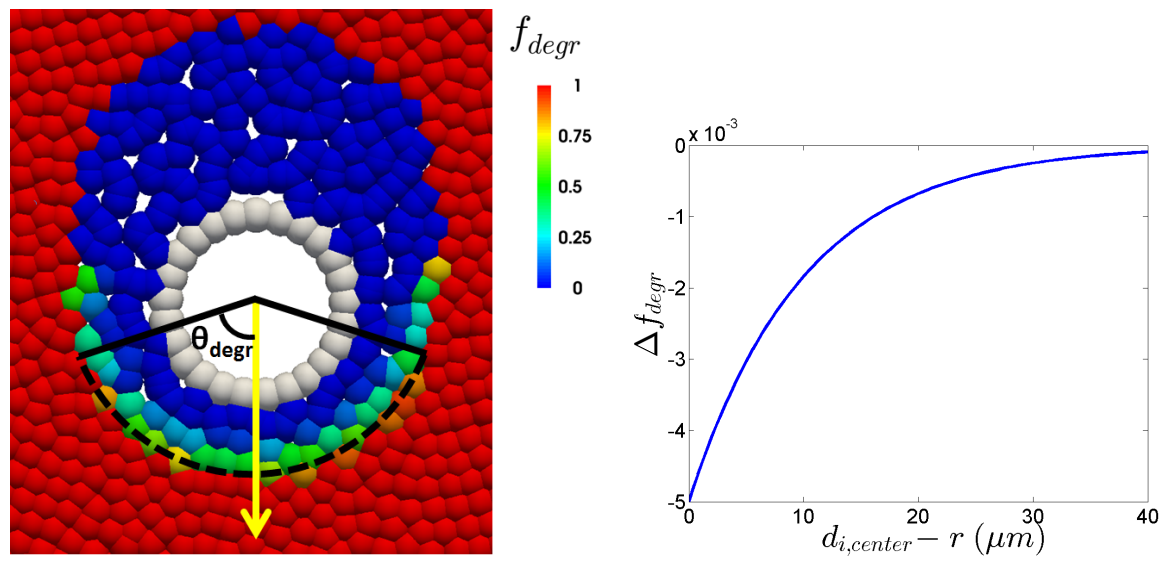

Figure 14: Left: Illustration of cell migration through the ECM by local ECM degradation. The cell migrates based on a migration force oriented in a certain direction (yellow arrow) and, after an initial degradation around the entire cell, material particles located within a region given by the degradation angle $\theta_{\text {degr }}$ from the migration force direction and within a maximal distance from the cell center are degraded by decreasing the degradation factor $f_{\text {degr }}$. Right: Example of the change in degradation factor $\Delta f_{\text {degr }}$ for one time step $d t$ as function of the distance to the cell boundary $\left(d_{i, \text { center }}-r\right)$ as described in Eq. 35 .

Besides, the buildup of deviatoric stress should only occur between solid particles and not when a fluid particle is moving relative to a solid particle. To ensure this the deviatoric stress part of the first term in the equation of momentum (Eq. (25p) and the formula for the rate of change of deviatoric stress (Eq. 26p) are multiplied by $\left(f_{\mathrm{degr}, i} f_{\mathrm{degr}, j}\right)$. In this way moving fluid particles can not lead to a buildup of deviatoric stress in the ECM. Third, the constant $\xi$ in the equation of state (Eq. (7) ) is chosen to be unaffected by degradation and thus remains 1 for all particles. This leads to a more compressible fluid around the cell after degradation, but avoids instabilities due to fast buildup of hydrostatic forces when $\xi$ would be allowed to increase upon degradation of solid particles. Finally, in order to prevent the rare occurrence of penetration of the boundary by a single particle, a repulsive force is added that pushes the particle away from the boundary when the distance to the boundary is closer 
than a threshold distance (here set at 0.7 times the interparticle distance).

A rigid circular cell with a radius of $40 \mu \mathrm{m}$ is placed in ECM, modeled as a rectangular domain $\left(\mathrm{E}=2.5 \mathrm{kPa}, \nu=0.45, \mu=100 \mathrm{~Pa} \cdot \mathrm{s}\right.$ and $\left.\rho_{0}=1000 \mathrm{~kg} / \mathrm{m}^{3}\right)$ of $600 \times 800 \mu \mathrm{m}$ with an interparticle distance of $10 \mu \mathrm{m}(\mathrm{h}=13.0 \mu \mathrm{m})$. The latter distance was found to guarantee resolution-independent results (see Appendix B). The particles are distributed using a method which iteratively uses weighted Voronoi tessellations to get an even distribution of particles in an arbitrary area [32]. In this way, preferred mesh directions are minimized and an even initial distribution around the cell is guaranteed, which is not the case for a cubic or hexagonal lattice. The Wendland kernel (see [25]) is used in this simulation to reduce particle clustering after degradation.

A migration force $\boldsymbol{F}_{\text {migr }}$ with a constant value $F_{\text {migr }}=10 \mu \mathrm{N}$ and a fluctuating direction representing Brownian motion is applied to the cell:

$$
\boldsymbol{F}_{\text {migr }}=F_{\text {migr }} \boldsymbol{u}_{\text {migr }}
$$

where $\boldsymbol{u}_{\text {migr }}$ is a unit vector in the direction of the migration force with a fluctuating angle $\theta_{\text {migr }}$ :

$$
\frac{\mathrm{d} \theta_{\text {migr }}}{\mathrm{d} t}=f_{\mathrm{bias}}\left(\theta_{\mathrm{migr}}^{\mathrm{bias}}-\theta_{\text {migr }}\right)+\sqrt{2 D_{r}} \zeta .
$$

This angle changes over time due to rotation towards a bias angle $\theta_{\text {migr }}^{\text {bias }}$ with a polarization rate $f_{\text {bias }}=0.1 \mathrm{~s}^{-1}$ and due to rotational noise with the rotational diffusion coefficient $D_{r}=5 \times 10^{-5} \mathrm{~s}^{-1}$ and Gaussian white noise $\zeta(t)$. We apply a force of $\boldsymbol{F}_{\text {migr }}=10 \mu \mathrm{N}$ on the cell, rather than a more realistic force value for the cell in the range of $\mathrm{pN}$ to a few $\mu \mathrm{N}$, in order to demonstrate a cell trajectory over a longer distance.

An initial degradation step is performed for all NSPH particles within $40 \mu \mathrm{m}$ of the cell boundary with a very high degradation rate $\frac{1}{\tau_{\operatorname{degr}}}=1.0 \times 10^{5} \mathrm{~s}^{-1}$ to generate a fluidized area around the cell. This allows the cell to move at the start of the simulation, which would otherwise be prevented by the no-slip boundary condition between the cell and the ECM. After this initial step, the degradation 
width of $40 \mu \mathrm{m}$ is maintained, but in combination with a degradation angle $\theta_{\text {degr }}$ of $90^{\circ}$ and a reference degradation rate of $\frac{1}{\tau_{\text {degr }}}=10 \mathrm{~s}^{-1}$. A simulation $\left(\operatorname{Re}=1.0 \times 10^{-7}\right)$ is run for $600 \mathrm{~s}$ with a time step of $0.01 \mathrm{~s}$. In Fig. 15 it can be seen that the cell has migrated through the ECM leaving behind a trail of degraded matrix with low von Mises stress caused by the local degradation. In front of the cell, the ECM is deformed resulting in a higher deviatoric stress. A supporting video of this simulation is attached in the online manuscript. In this simulation the cell reaches an average velocity of $48 \mu \mathrm{m} / \mathrm{min}$ which, as expected by the high migration force applied, was higher than reported migration forces for embedded cells in literature. Raeber et al. for example reported migration velocities of approximately $0.1-0.3 \mu \mathrm{m} / \mathrm{min}$ for fibroblasts in biodegradable PEG hydrogels [33. 


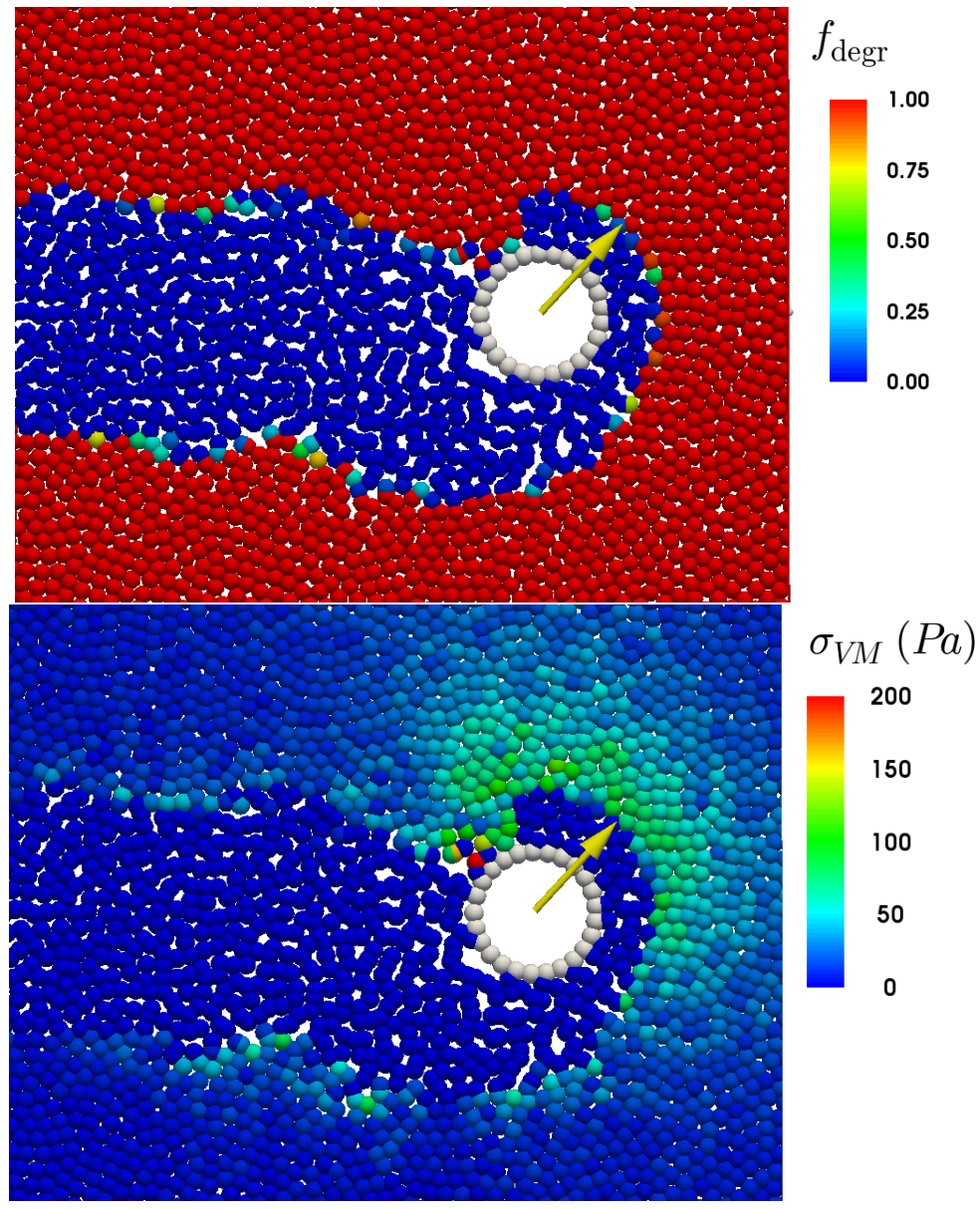

Figure 15: Degradation factor $f_{\mathrm{degr}}$ (top) and von Mises stress $\boldsymbol{\sigma}_{\mathrm{VM}}$ distribution (bottom) in the degradable viscoelastic ECM around a migrating cell. The cell degrades the ECM by fluidizing the viscoelastic material and leaves behind a trail of degraded material. The ECM in front of the migrating cell is deformed and builds up deviatoric stress.

Next, in order to demonstrate the effect of degradation rate on cell migration speed, 16 simulations are performed with values of reference degradation rates $\frac{1}{\tau_{\text {degr }}}$ ranging from 0.002 to $200 \mathrm{~s}^{-1}$. The migration speed as a function of degradation rate is shown in Fig. 16. It can be seen that the cell migration speed follows a sigmoid curve with a plateau of low migration speed at low degradation rates, an increase in migration rate at intermediate migration speeds and 
a second plateau of maximal migration at high degradation rates. This is in agreement with our basic understanding that increased ECM degradation can not monotonically augment cell migration.

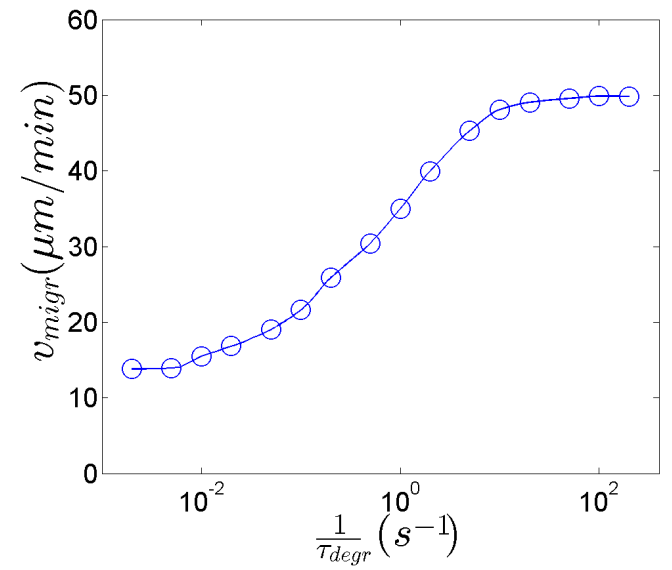

Figure 16: Cell migration speed in $\mu \mathrm{m} / \mathrm{min}$ as function of degradation rate $\frac{1}{\tau_{\mathrm{degr}}}$ in $\mathrm{s}^{-1}$ for migration of a circular cell through a degradable viscoelastic ECM. The migration speed follows a sigmoid curve with a plateau of low cell migration speed for low degradation rate and a plateau of high cell migration speed for high degradation rate. 

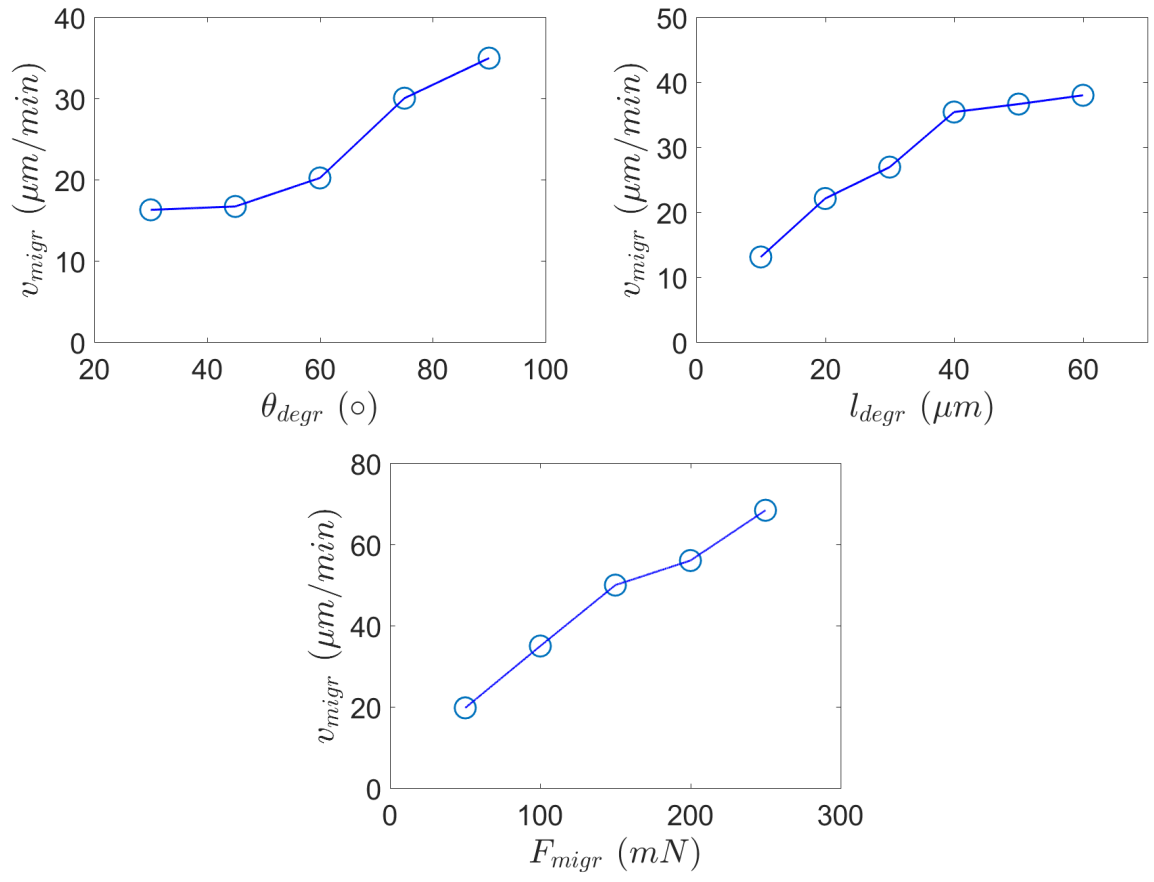

Figure 17: Migration velocity as function of degradation angle $\theta_{\text {degr }}$ (top, left), degradation width $l_{\text {degr }}$ (top, right) and migration force $F_{m i g r}$ (bottom) for migration of a circular cell through a degradable viscoelastic ECM.

In order to demonstrate the sensitivity of the migration model, a parameter study is performed in which the influence of 3 migration parameters on migration velocity is investigated. The results of this study are shown in Figure 17. First, when the degradation angle $\theta_{\text {degr }}$ is decreased from $90^{\circ}$ to $30^{\circ}$, the migration velocity of the cell reduces until it reaches a velocity similar to the velocity observed for very low degradation rates in Figure 16. This result is expected as a decreasing $\theta_{\text {degr }}$ leads to a smaller path of degraded ECM and eventually prevents degradation completely. Second, the width of the degradation zone $\left(l_{\text {degr }}\right)$ is varied, while keeping the degradation rate for particles in this zone unchanged. The initial degradation step for all particles within $40 \mu \mathrm{m}$ of the cell boundary was maintained. It can be seen that an increased degradation width results in an increased migration velocity until a width of $40 \mu \mathrm{m}$ after 
which a further increase of the degradation width does not affect the migration velocity. This is expected as the degradation rate is relatively small at a distance further away than $40 \mu \mathrm{m}$ from the cell boundary (see Figure 14). Finally, the migration force $F_{m i g r}$ is varied. As expected, an increase in migration force results in a higher migration velocity. For the migration force values chosen here the migration velocity at higher migration forces appears to be not limited by the degradation rate.

\section{Discussion}

In this paper, smoothed particle hydrodynamics was proposed as a method for modeling cell-ECM interactions. In order to achieve accurate hydrostatic pressure and stress profiles in the ECM around a cell, we applied the corrected boundary conditions in SPH as proposed by Kulasegaram et al. and Ferrand et al. 22, 23 and extended these boundary conditions so that they can be used for moving boundaries in contact with a viscoelastic solid. In addition, a contact criterion was implemented, leading to an accurate calculation of hydrostatic pressures and deviatoric stresses during contact establishment as could be assessed by comparison to FE simulations. By performing benchmark simulations the effectiveness and pitfalls of our extended method were demonstrated. As was shown in Section 3.4, this method is able to capture important characteristics of cell migration such as ECM degradation, large deformations of the ECM and the migration of a cell through the ECM in a mechanically sound manner. To the best of our knowledge, a model, either mesh-based or meshless, that captures cell migration through a continuous ECM that functions as a mechanical obstacle for the cell and requires degradation for the cell to pass through, has not been published before.

First, NSPH, a variant of SPH where the equations of motion are assumed to be overdamped, was introduced as a method to model the viscoelastic behavior of the ECM in a low Reynolds number environment. When a force was applied to circular regions, that could represent focal adhesions when simulated at the 
$\mu \mathrm{m}$-scale, viscoelastic NSPH was able to reproduce the results obtained by viscoelastic SPH as well as FE both transiently and at equilibrium (see Section 3.1). While SPH required $4 \times 10^{6}$ time steps to reach the equilibrium solution, NSPH only needed 100 time steps, thus demonstrating the computational efficiency of NSPH. Besides, for a similar simulation at the $\mu \mathrm{m}$-scale the time step for SPH would become prohibitively small, as it decreases quadratically with the particle spacing (see Eq. (34)). In contrast, the time step for NSPH will not change, as it is independent of particle spacing. Therefore, for simulations at the cell scale, because of computational costs, the use of NSPH is clearly preferred.

Next, the boundary condition proposed by Kulasegaram et al. and Ferrand et al. [22, 23] was introduced and extended to model the contact between a viscous fluid or viscoelastic solid modeled by means of NSPH and a rigid, yet moving object. In Section 3.2 it was shown that for fluid flow around an array of rigid and stationary square pillars the extended boundary method leads to results similar to those for a rigid square filled with particles, both for SPH and NSPH. Besides, NSPH reaches the equilibrium solution after 10 time steps, while SPH reaches the same equilibrium solution after 10000 time steps. Taken together, these results demonstrate the accuracy and efficiency of NSPH and the extended boundary method for modeling low Reynolds number flow around rigid boundaries.

When an object approaches a material that is modeled by means of (N)SPH the material starts to build up pressure and deviatoric stress as soon as the object enters the kernel support [22, 23]. However, the pressure and deviatoric stress inside a material should be zero when all particles are at exactly one interparticle distance from their closest neighbors. Therefore, the pressure and deviatoric stress should only start to build up when the material is within one interparticle distance from the boundary. In order to ensure this, a contact criterion was proposed. In Section 3.3 the importance of this contact criterion, in combination with the extended boundary method was demonstrated for the indentation of a viscoelastic solid with a rigid, yet moving circular indenter. For 
SPH and NSPH simulations that made use of the contact criterion, hydrostatic pressure and von Mises stress distributions similar to FE were obtained. The contact criterion strongly reduced spurious hydrostatic pressures and von Mises stresses that were encountered in simulations without any contact criterion. The proposed contact criterion could prove to be problematic when an object approaches an SPH material with a large velocity as contact is only established when the object is within one interparticle distance from the material. This would require the use of a lower time step. Fortunately, movements of cells are relatively slow and are not expected to cause large problems for contact detection when the contact criterion is used.

Finally, cell migration through a viscoelastic ECM was simulated in order to illustrate the potential of the proposed meshless method to deal with cell-matrix interaction, including matrix degradation. It was shown that the cell leaves behind a trail of degraded matrix with low von Mises stress caused by the local degradation. The formation of such trails has been observed in experiments by for example Schultz et al. who measured by means of multiple particle tracking rheology how human mesenchymal stem cells remodel PEG hydrogels and who observed the formation of irreversible tracks in the PEG hydrogel as a result of degradation-mediated cell migration [34. When degradation rates are varied a sigmoid curve is observed for cell migration speed as a function of degradation rate with a minimal speed for low degradation rate and a plateau of maximal speed for high degradation rates. It should be highlighted that representation of a cell as a rigid sphere is a big simplification and that these simulations merely illustrate the potential of this method to deal with cell-matrix interactions during cell migration. In the future, more realistic simulations of cell migration can be envisioned by replacing the rigid cell with a deformable cell that captures the viscoelastic properties of the cell cortex as in 13]. The addition of filopodia-like structures as in [2] that enable the cell to protrude, adhere and apply local pulling forces could replace the migration force that was used here to render the cell its motility.

In conclusion, we have proposed and validated a new computational method 
for modeling of a viscoelastic ECM by means of SPH. Contact between ECM and a cell is modeled by extending an existing boundary method such that it can handle contact between a viscoelastic material and a rigid, yet moving boundary. A contact criterion is introduced to reduce errors in the calculation of hydrostatic pressure and deviatoric stress at the cell-ECM interface. By adding local degradation this method is able to capture some qualitative aspects of cell migration through an ECM, which can later be extended towards more realistic cell-ECM interactions.

\section{Acknowledgments}

The authors thank FWO for the research grant G0821.13. T.H. is a $\mathrm{PhD}$ fellow of FWO-Vlaanderen. H.V.O. acknowledges that the research leading to these results has received funding from the European Research Council under the European Union's Seventh Framework Programme (FP7/2007-2013)/ ERC Grant Agreement $n^{\circ}$ 308223). B.S. acknowledges the Agency for Innovation by Science and Technology in Flanders (IWT). P.V.L. greatly acknowledges the project INVADE (INSERM, France).

[1] B. Lee, X. Zhou, K. Riching, K. W. Eliceiri, P. J. Keely, S. A. Guelcher, A. M. Weaver, Y. Jiang, A three-dimensional computational model of collagen network mechanics, PLoS ONE 9 (11) (2014) e111896. doi: 10.1371/journal.pone.0111896.

[2] M.-C. Kim, J. Whisler, Y. R. Silberberg, R. D. Kamm, Cell invasion dynamics into a three dimensional extracellular matrix fibre network, PLoS computational biology 11 (10) (2015) e1004535. doi:10.1371/journal. pcbi.1004535.

[3] J. Zhu, A. Mogilner, Comparison of cell migration mechanical strategies in three-dimensional matrices: a computational study., Interface focus 6 (5) (2016) 20160040. doi:10.1098/rsfs. 2016.0040

URL http://www.ncbi.nlm.nih.gov/pubmed/27708764 
[4] T. C. Gasser, R. W. Ogden, G. A. Holzapfel, Hyperelastic modelling of arterial layers with distributed collagen fibre orientations., Journal of the Royal Society, Interface / the Royal Society 3 (6) (2006) 15-35. arXiv: 0312002v1, doi:10.1098/rsif.2005.0073

[5] D. Zeng, A. Ferrari, J. Ulmer, A. Veligodskiy, P. Fischer, J. Spatz, Y. Ventikos, D. Poulikakos, R. Kroschewski, Three-dimensional modeling of mechanical forces in the extracellular matrix during epithelial lumen formation, Biophysical Journal 90 (12) (2006) 4380-4391. doi:10.1529/ biophysj.105.073494.

URL http://dx.doi.org/10.1529/biophysj.105.073494

[6] R. Zielinski, D. Kniss, Finite element analysis of traction force microscopy: influence of cell mechanics, adhesion , and morphology, Journal of Biomechanical Engineering 135 (7) (2013) 71009. doi:10.1115/1.4024467

[7] T. A. M. Heck, W. Wilson, J. Foolen, A. C. Cilingir, K. Ito, C. C. V. Donkelaar, A tissue adaptation model based on strain-dependent collagen degradation and contact-guided cell traction, Journal of Biomechanics 48 (5) (2015) 823-831. doi:10.1016/j.jbiomech.2014.12.023.

URL http://dx.doi.org/10.1016/j.jbiomech.2014.12.023

[8] J. A. Mackenzie, M. Nolan, R. H. Insall, Local modulation of chemoattractant concentrations by single cells: dissection using a bulksurface computational model Interface Focus 6 (5) (2016) 20160036. doi:10.1098/rsfs.2016.0036.

URL http://rsfs.royalsocietypublishing.org/content/6/5/ 20160036

[9] Y. Guyot, B. Smeets, T. Odenthal, R. Subramani, F. P. Luyten, H. Ramon, I. Papantoniou, L. Geris, Immersed Boundary Models for Quantifying Flow-Induced Mechanical Stimuli on Stem Cells Seeded on 3D Scaffolds in Perfusion Bioreactors, PLoS Computational Biology 12 (9) (2016) e1005108. doi:10.1371/journal.pcbi.1005108. 
[10] S. E. Hieber, P. Koumoutsakos, A Lagrangian particle method for the simulation of linear and nonlinear elastic models of soft tissue, Journal of Computational Physics 227 (2008) 9195-9215. doi:10.1016/j.jcp.2008. 05.016 .

[11] S. M. Hosseini, J. J. Feng, A particle-based model for the transport of erythrocytes in capillaries, Chemical Engineering Science 64 (22) (2009) 4488-4497. doi:10.1016/j.ces.2008.11.028. URL http://dx.doi.org/10.1016/j.ces.2008.11.028

[12] P. Van Liedekerke, P. Ghysels, E. Tijskens, G. Samaey, B. Smeets, D. Roose, H. Ramon, A particle-based model to simulate the micromechanics of single-plant parenchyma cells and aggregates, Physical Biology 7 (2) (2010) 026006. doi:10.1088/1478-3975/7/2/026006.

[13] T. Odenthal, B. Smeets, P. Van Liedekerke, E. Tijskens, H. Van Oosterwyck, H. Ramon, Analysis of initial cell spreading using mechanistic contact formulations for a deformable cell model, PLoS Computational Biology 9 (10) (2013) e1003267. doi:10.1371/journal.pcbi.1003267. URL http://www.pubmedcentral.nih.gov/articlerender.fcgi? artid=3798278\{\&\}tool=pmcentrez $\{\&\}$ rendertype=abstract

[14] R. A. Gingold, J. J. Monaghan, Smoothed particle hydrodynamics - Theory and application to non-spherical stars, Montly Notices of the Royal Astronomical Society 171 (1977) 375-389.

[15] L. B. Lucy, A numerical approach to the testing of the fission hypothesis, Astronomical Journal 82 (1977) 1013-1020.

[16] L. Di G. Sigalotti, J. Klapp, O. Rendón, C. A. Vargas, F. Peña-Polo, On the kernel and particle consistency in smoothed particle hydrodynamics, Applied Numerical Mathematics 108 (2016) 242-255. arXiv:1605.05245, doi:10.1016/j.apnum.2016.05.007. 
[17] R. Das, P. W. Cleary, Evaluation of Accuracy and Stability of the Classical SPH Method Under Uniaxial Compression, Journal of Scientific Computing 64 (3) (2015) 858-897. doi:10.1007/s10915-014-9948-4.

URL http://dx.doi.org/10.1007/s10915-014-9948-4

[18] P. Van Liedekerke, B. Smeets, T. Odenthal, E. Tijskens, H. Ramon, Solving microscopic flow problems using Stokes equations in SPH, Computer Physics Communications 184 (7) (2013) 1686-1696. doi:10.1016/j.cpc.2013.02.013

URL http://linkinghub.elsevier.com/retrieve/pii/ S0010465513000702

[19] P. Van Liedekerke, M. Palm, N. Jagiella, D. Drasdo, Simulating tissue mechanics with Agent Based Models : concepts and perspectives, Computational Particle Mechanics 2 (4) (2015) 401-444.

[20] J. Gray, J. Monaghan, R. Swift, SPH elastic dynamics, Computer Methods in Applied Mechanics and Engineering 190 (49-50) (2001) 6641-6662.

[21] L. Lobovský, J. Kren, Smoothed particle hydrodynamics modelling of fluids and solids, Applied and Computational Mechanics 1 (2007) 521-530.

[22] M. Ferrand, D. R. Laurence, B. D. Rogers, D. Violeau, C. Kassiotis, Unified semi-analytical wall boundary conditions for inviscid, laminar or turbulant flows in the meshless SPH method, International Journal for numerical methods in fluids 71 (2013) 446-472.

[23] S. Kulasegaram, J. Bonet, R. W. Lewis, M. Profit, A variational formulation based contact algorithm for rigid boundaries in two-dimensional SPH applications, Computational Mechanics 33 (4) (2004) 316-325. doi: $10.1007 / \mathrm{s} 00466-003-0534-0$

[24] J. Monaghan, Smoothed particle hydrodynamics, Reports on Progress in Physics 68 (2005) 1703-1759. doi:10.1088/0034-4885/68/8/R01 
[25] H. Wendland, Piecewise polynomial, positive definite and compactly supported radial functions of minimal degree, Advances in Computational Mathematics 4 (1) (1995) 389-396.

[26] J. P. Morris, P. J. Fox, Y. Zhu, Modeling low Reynolds number incompressible flows using SPH, Journal of Computational Physics 136 (1) (1997) 214-226. doi:10.1006/jcph.1997.5776.

URL http://linkinghub.elsevier.com/retrieve/pii/ S0021999197957764

[27] J. Monaghan, Simulating free surface flows with SPH, Journal of Computational Physics 110 (1994) 399-406.

[28] M. T. Heath, Scientific computing: an introductory survey, 2nd Edition, McGraw-Hill, 2005.

[29] A. Leroy, D. Violeau, M. Ferrand, C. Kassiotis, Unified semi-analytical wall boundary conditions applied to 2-D incompressible SPH, Journal of Computational Physics 261 (2014) 106-129. doi:10.1016/j.jcp.2013. 12.035

URL http://dx.doi.org/10.1016/j.jcp.2013.12.035

[30] D. K. Schlüter, I. Ramis-Conde, M. A. J. Chaplain, Computational modeling of single-cell migration: The leading role of extracellular matrix fibers, Biophysical Journal 103 (6) (2012) 1141-1151. doi:10.1016/j.bpj.2012. 07.048 .

[31] S. J. Mousavi, M. Doblaré, M. H. Doweidar, Computational modelling of multi-cell migration in a multi-signalling substrate., Physical biology 11 (2) (2014) 026002. doi:10.1088/1478-3975/11/2/026002.

URL http://www.ncbi.nlm.nih.gov/pubmed/24632566

[32] S. Diehl, G. Rockefeller, C. L. Fryer, D. Riethmiller, T. S. Statler, Generating optimal initial conditions for smoothed particle hydrodynamics simulations, arXiv:1211.0525 [astro-ph.IM]arXiv:arXiv:1211.0525v2. 
[33] G. P. Raeber, M. P. Lutolf, J. a. Hubbell, Mechanisms of 3-D migration and matrix remodeling of fibroblasts within artificial ECMs, Acta biomaterialia 3 (5) (2007) 615-629. doi:10.1016/j.actbio.2007.03.013.

URL http://www.ncbi.nlm.nih.gov/pubmed/17572164

[34] K. M. Schultz, K. A. Kyburz, K. S. Anseth, Measuring dynamic cell material interactions and remodeling during 3D human mesenchymal stem cell migration in hydrogels, Proceedings of the National Academy of Sciences of the United States of America 112 (29) (2015) E3757-E3764. doi:10.1073/pnas.1511304112.

[35] Abaqus, Viscoelastic rod subjected to constant axial load. Abaqus Documentation - Abaqus Benchmarks Guide, section 3.1.1.

\section{Appendices}

\section{A. Modeling of a Kelvin-Voigt solid using finite element method}

The current viscoelastic NSPH implementation defines essentially a KelvinVoigt material, for which the constitutive relationship is given by:

$$
\boldsymbol{\sigma}^{\mathrm{hyd}}(t)=3 K \boldsymbol{\epsilon}^{\mathrm{vol}}(t)+3 \lambda \frac{\mathrm{d} \boldsymbol{\epsilon}^{\mathrm{vol}}(t)}{\mathrm{d} t}
$$

and

$$
\boldsymbol{\sigma}^{\mathrm{dev}}(t)=2 G \boldsymbol{\epsilon}^{\mathrm{dev}}(t)+2 \mu \frac{\mathrm{d} \boldsymbol{\epsilon}^{\mathrm{dev}}(t)}{\mathrm{d} t},
$$

where $\boldsymbol{\sigma}^{\text {hyd }}$ and $\boldsymbol{\sigma}^{\text {dev }}$ are the hydrostatic and deviatoric stresses, $\boldsymbol{\epsilon}^{\text {hyd }}$ and $\boldsymbol{\epsilon}^{\text {dev }}$ are the volumetric and deviatoric strains, $K$ and $\lambda$ are the bulk modulus and bulk viscosity and $G$ and $\mu$ are the shear modulus and shear viscosity respectively. It is not possible to directly model a Kelvin-Voigt material in Abaqus, since the infinitely large instantaneous modulus and the infinitely small relaxation time can not be prescribed. However, it is possible to approximate the 
Kelvin-Voigt material by appropriately selecting the relaxation factor (that relates instantaneous and equilibrium moduli) and the relaxation time constant. If the Young's modulus and Poisson's ratio are defined as $E$ and $\nu$, then the bulk and shear moduli can be determined respectively as:

$$
K=\frac{E}{3(1-2 \nu)}
$$

and

$$
G=\frac{E}{2(1+\nu)} .
$$

In a 2D formulation, Morris et al. 24] state that the bulk modulus is given by

$$
\lambda=\frac{5 \mu}{3} .
$$

Taking the Laplace transform of the constitutive relationships results in:

$$
\boldsymbol{\Sigma}^{\mathrm{hyd}}(s)=3 K \boldsymbol{\varepsilon}^{\mathrm{vol}}(s)+3 \lambda s \varepsilon^{\mathrm{vol}}(s)
$$

and

$$
\Sigma^{\operatorname{dev}}(s)=2 G K \varepsilon^{\operatorname{dev}}(s)+2 \mu s \varepsilon^{\operatorname{dev}}(s),
$$

where $\boldsymbol{\Sigma}$ and $\boldsymbol{\varepsilon}$ denote the Laplace transformed functions of $\boldsymbol{\sigma}$ and $\boldsymbol{\epsilon}$ respectively and $s$ is the Laplace transform variable. Substituting Eq. 41, , 42 and 43 in the Laplace transform functions above gives:

$$
\Sigma^{\mathrm{hyd}}=\frac{E \varepsilon^{\mathrm{vol}}}{1-2 \nu}+5 \mu s \varepsilon^{\mathrm{vol}}
$$

and

$$
\boldsymbol{\Sigma}^{\mathrm{dev}}=\frac{E \varepsilon^{\mathrm{dev}}}{1+\nu}+2 \mu s \varepsilon^{\mathrm{dev}},
$$

where for simplicity the dependence on $s$ has not been written explicitly. Eq. 46) and 47) can be simplified as:

$$
\varepsilon^{\mathrm{vol}}=\frac{1-2 \nu}{E} \frac{\boldsymbol{\Sigma}^{\mathrm{hyd}}}{1+s \tau_{K}}
$$

and

$$
\varepsilon^{\mathrm{dev}}=\frac{1+\nu}{E} \frac{\boldsymbol{\Sigma}^{\mathrm{dev}}}{1+s \tau_{G}}
$$


with the following time constants:

$$
\tau_{K}=\frac{\lambda}{K}=\frac{5 \mu(1-2 \nu)}{E}
$$

and

$$
\tau_{G}=\frac{\mu}{G}=\frac{2 \mu(1+\nu)}{E} .
$$

In a creep test, the applied stress is given by:

$$
\boldsymbol{\sigma}=\boldsymbol{\sigma}^{0} H(t)
$$

where $H(t)$ is the unit step function. The Laplace transform of this equation is given by:

$$
\frac{\boldsymbol{\sigma}^{0}}{s}
$$

The Laplace transformed equations with $\boldsymbol{\Sigma}^{\text {hyd }}=\sigma^{0, \text { hyd }}$ and $\boldsymbol{\Sigma}^{\text {dev }}=\sigma^{0, \text { dev }}$ gives:

$$
\varepsilon^{\mathrm{vol}}=\frac{1-2 \nu}{E} \frac{\boldsymbol{\sigma}^{0, \mathrm{hyd}}}{s\left(1+s \tau_{K}\right)}=\frac{1-2 \nu}{E} \frac{\boldsymbol{\sigma}^{0, \mathrm{hyd}}}{\frac{1}{s}-\frac{\tau_{K}}{1+s \tau_{K}}}
$$

and

$$
\varepsilon^{\mathrm{dev}}=\frac{1+\nu}{E} \frac{\boldsymbol{\sigma}^{0, \mathrm{dev}}}{s\left(1+s \tau_{G}\right)}=\frac{1+\nu}{E} \frac{\boldsymbol{\sigma}^{0, \mathrm{dev}}}{\frac{1}{s}-\frac{\tau_{G}}{1+s \tau_{G}}} .
$$

Taking the inverse Laplace transforms gives:

$$
\boldsymbol{\epsilon}^{\mathrm{vol}}(t)=\frac{1-2 \nu}{E} \boldsymbol{\sigma}^{0, \mathrm{hyd}}\left(1-e^{-\left(\frac{t}{\tau_{K}}\right)}\right)
$$

and

$$
\boldsymbol{\epsilon}^{\mathrm{dev}}(t)=\frac{1+\nu}{E} \boldsymbol{\sigma}^{0, \operatorname{dev}}\left(1-e^{-\left(\frac{t}{\tau_{G}}\right)}\right) .
$$

The above expressions can be recovered from the following approximations in the limit of a very small number $\delta<\langle 1$ :

$$
\boldsymbol{\epsilon}^{\mathrm{vol}}(t) \frac{1-2 \nu}{E} \boldsymbol{\sigma}^{0, \text { hyd }}\left(1-(1-\delta) e^{-\left(\frac{t}{1-(\delta) \tau_{K}}\right)}-\delta e^{-\left(\frac{t}{1-(\delta) \tau_{G}}\right)}\right)
$$


and

$$
\boldsymbol{\epsilon}^{\operatorname{dev}}(t) \frac{1+\nu}{E} \boldsymbol{\sigma}^{0, \operatorname{dev}}\left(1-\delta e^{-\left(\frac{t}{1-(\delta) \tau_{K}}\right)}-(1-\delta) e^{-\left(\frac{t}{1-(\delta) \tau_{G}}\right)}\right) .
$$

This approximated response can be fitted using the Prony series [35]:

$$
G(t)=G_{0}\left(1-g_{1}\left(1-e^{-\left(\frac{t}{\tau_{1}}\right)}\right)-g_{2}\left(1-e^{-\left(\frac{t}{\tau_{2}}\right)}\right)\right)
$$

and

$$
K(t)=K_{0}\left(1-k_{1}\left(1-e^{-\left(\frac{t}{\tau_{1}}\right)}\right)-k_{2}\left(1-e^{-\left(\frac{t}{\tau_{2}}\right)}\right)\right)
$$

with:

$$
\begin{gathered}
\tau_{1}=\tau_{K} \delta=5 \mu \delta \frac{1-2 \nu}{E} \\
\tau_{2}=\tau_{G} \delta=2 \mu \delta \frac{1+\nu}{E}
\end{gathered}
$$

$$
g_{1}=\delta
$$

$$
g_{2}=1-2 \delta,
$$

$$
k_{1}=1-2 \delta,
$$

$$
k_{2}=\delta
$$

$$
G_{0}=\frac{G}{1-g_{1}-g_{2}}=\frac{E}{2 \delta(1+\nu)}
$$

and

$$
K_{0}=\frac{K}{1-k_{1}-k_{2}}=\frac{E}{3 \delta(1-2 \nu)} .
$$

By taking $\delta=0.1$ and the material properties similar to the (N)SPH simulations in Section 3.1, i.e. $E=2.5 \mathrm{~Pa}, \nu=0.45$ and $\mu=100 \mathrm{~Pa} \cdot \mathrm{s}$, the parameters in 
the equations can be calculated to be: $\tau_{1}=2 \mathrm{~s}, \tau_{2}=11.6 \mathrm{~s}, g_{1}=0.1, g_{2}=0.8$, $k_{1}=0.8, k_{2}=0.1, G_{0}=8.62 \mathrm{~Pa}$ and $K_{0}=83.33 \mathrm{~Pa}$, resulting in $E_{0}=25 \mathrm{~Pa}$ and $\nu_{0}=0.45$.

The simulation is executed in two steps. In the first step, the body forces are applied to the selected circular regions by linear increase of the force magnitude over a period of $0.1 \mathrm{~s}$. In the second step, the body forces are held fixed over a time period of $1000 \mathrm{~s}$, with a time increment size varying between $0.2 \mathrm{~s}$ and 20 s. The results of this simulation are shown in Fig. 7 and compared with both SPH and NSPH.

\section{B. Effect of resolution on degradation-mediated cell migration simu- lation}

Two simulations are performed to demonstrate the effect of resolution for the degradation-mediated cell migration simulation in Section 3.4 For these simulations, that run for $200 \mathrm{~s}$, a smaller rectangular domain of $300 \times 400 \mu \mathrm{m}$ is used. One simulation is run with an interparticle distance of $10 \mu \mathrm{m}$ and the other simulation with an interparticle distance of $5 \mu \mathrm{m}$. The von Mises stress distribution after $200 \mathrm{~s}$ can be seen in Fig. 18 for both simulations. It can be observed that the von Mises stress distribution is similar for both resolutions. In Fig. 19 the migration path for the cell center in both simulations is shown. It can be seen that in both simulations the cell follows a similar path with a distance between the cell center after $200 \mathrm{~s}$ of only $2.76 \mu \mathrm{m}$ (while the radius of the cell is $40 \mu \mathrm{m})$. These results demonstrate that the coupling of viscoelastic NSPH with the extended boundary method works well for different resolutions and that the resolution of the simulations performed in Section 3.4 is high enough to ensure resolution-independent results. 


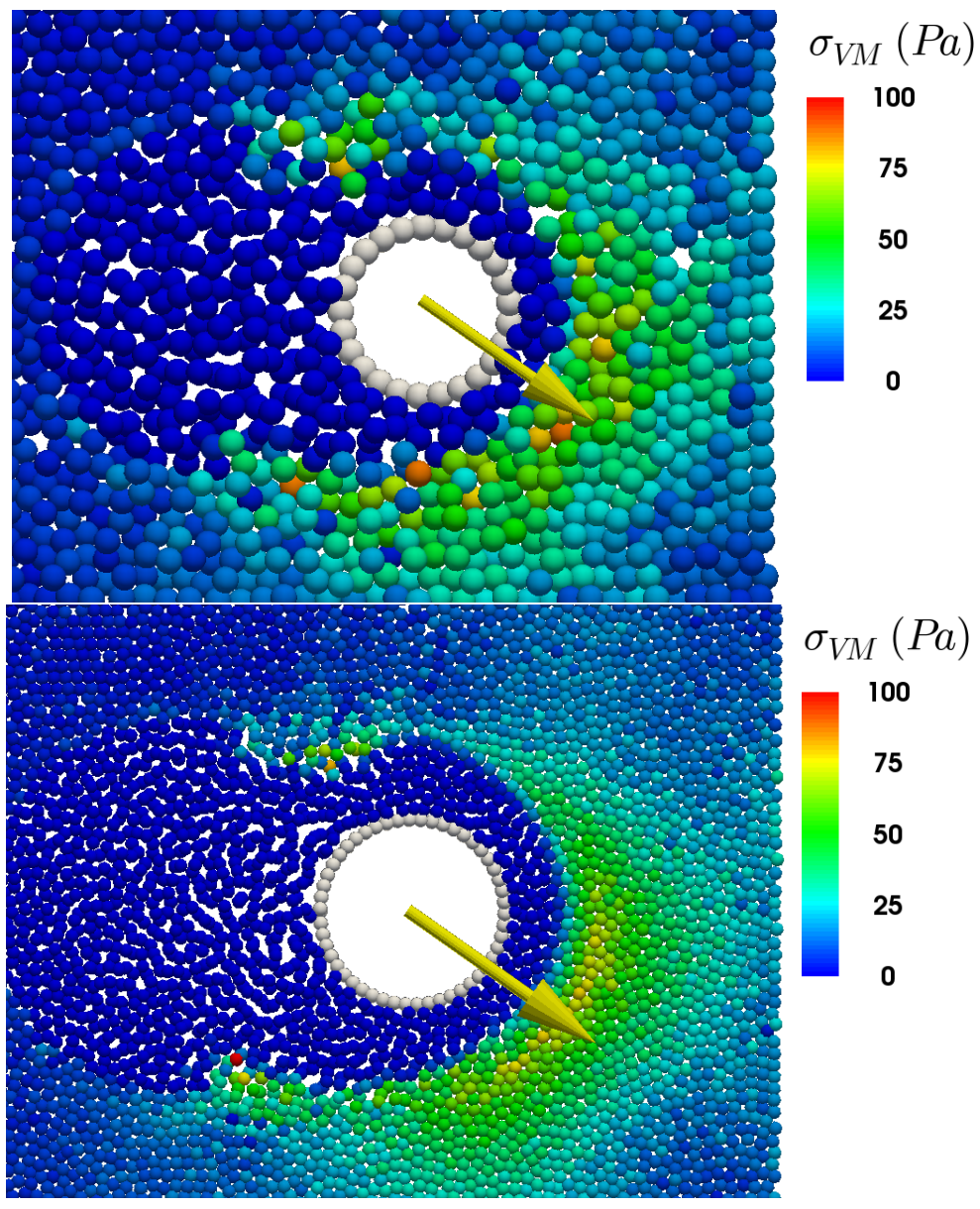

Figure 18: Von Mises stress $\boldsymbol{\sigma}_{\mathrm{VM}}$ in the degradable viscoelastic ECM around a migrating cell for a simulation with low (top) and high (bottom) resolution. The cell degrades the ECM by fluidizing the viscoelastic material and leaves behind a trail of degraded material. The ECM in front of the migrating cell is deformed and builds up deviatoric stress. 


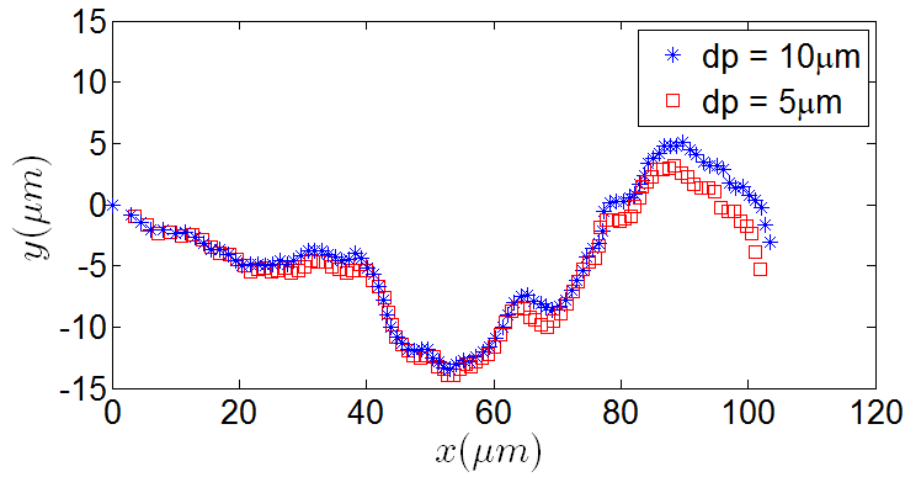

Figure 19: Cell center migration path for a degradation-mediated cell migration simulation with a low resolution $(*)$ and a high resolution $(\square)$. Both cells start in the origin and the same fluctuating migration force is applied. The distance between both cell centers after $200 \mathrm{~s}$ of migration is $2.76 \mu \mathrm{m}$. Notice that the $\mathrm{y}$-axis is scaled compared to the $\mathrm{x}$-axis to better appreciate the path differences in the y-direction. 